\title{
Pathogenesis of Intradermal Staphylococcal Infections
}

\section{Rabbit Experimental Approach to Natural Staphylococcus aureus Skin Infections}

\author{
Asunción Muñoz-Silvestre, * Mariola Penadés, ${ }^{*}$ Laura Selva, * Sara Pérez-Fuentes, * Elena Moreno-Grua, * Ana García-Quirós, * \\ Juan J. Pascual, ${ }^{\dagger}$ Alberto Arnau-Bonachera, ${ }^{*}$ Agustín Barragán, ${ }^{*}$ Juan M. Corpa, ${ }^{*}$ and David Viana*
}

From the Biomedical Research Institute (PASAPTA-Pathology Group), * Facultad de Veterinaria, Universidad Cardenal Herrera-CEU, CEU Universities, Valencia; and the Institute for Animal Science and Technology, ${ }^{\dagger}$ Universitat Politècnica de València, Valencia, Spain

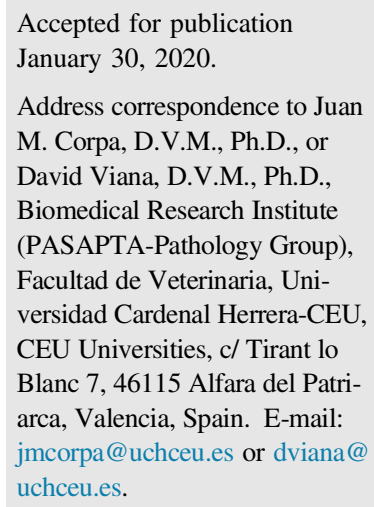

\begin{abstract}
Despite the enormous efforts made to achieve effective tools that fight against Staphylococcus aureus, the results have not been successful. This failure may be due to the absence of truly representative experimental models. To overcome this deficiency, the present work describes and immunologically characterizes the infection for 28 days, in an experimental low-dose (300 colony-forming units) intradermal model of infection in rabbits, which reproduces the characteristic staphylococcal abscess. Surprisingly, when mutant strains in the genes involved in virulence ( $\mathrm{J} \Delta a g r, \mathrm{~J} \Delta \operatorname{coa} \Delta v w b, \mathrm{~J} \Delta h l a$, and $\mathrm{J} \Delta p s m \alpha$ ) were inoculated, no strong effect on the severity of lesions was observed, unlike other models that use high doses of bacteria. The inoculation of a human rabbitized ( $F d l\left(t B^{r}\right)$ strain demonstrated its capacity to generate a similar inflammatory response to a wild-type rabbit strain and, therefore, validated this model for conducting these experimental studies with human strains. To conclude, this model proved reproducible and may be an option of choice to check both wild-type and mutant strains of different origins. (Am J Pathol 2020, 190: 1188-1210; https://doi.org/10.1016/ j.ajpath.2020.01.019)
\end{abstract}

Staphylococcus aureus is a widespread bacterium that has adapted well to humans and animals, which, if provided with a suitable opportunity, can initiate severe infections at various body sites. ${ }^{1-3}$ It can cause a wide range of diseases and syndromes, but the most worrying ones, given community-associated infections, are those that affect both skin and soft tissues. ${ }^{4-9}$ Skin and soft tissue infections can be minor and self-limiting, ${ }^{1}$ but if they become complicated, they can prove life-threatening and be characterized by the formation of large abscesses. ${ }^{10}$

After years of study and millions of dollars invested in research, gaps in our understanding of $S$. aureus infections remain, and it is not known in detail how staphylococcal infections evolve in humans. Numerous studies have been conducted on the pathogenesis of this bacterium, ${ }^{1}$ usually for the ultimate objective of developing therapeutic tools against staphylococcal infections. ${ }^{11}$ However, practical results remain frustrating because even today there are no effective preventive therapies, but resistance to antibiotics is growing and has reached really worrying levels. The problem could lie in the fact that the results experimentally obtained in inadequate animal models have been assumed and almost never questioned.

Supported by the Spanish Ministry of Economy and Competitiveness (MINECO) grant AGL2014-53405-C2-2-P, the Universidad CEU Cardenal Herrera, and European Union Development Fund (FEDER) Programme PO FEDER 2007-2013. A.M.-S., A.G.-Q., and E.M.-G. receive fellowship support from the Universidad CEU Cardenal Herrera; S.P.-F. receives fellowship support from the Generalitat Valenciana ACIF/2016/085; and M.P. and E.M.-G. receive fellowship support from the Spanish Ministry of Education, Culture, and Sport AP2010-3907 and FPU17/02708, respectively.

Disclosures: None declared. 
There are two important aspects to be taken into account in experimental staphylococcal infections: the animal species and the strain used. The murine has been the most frequently studied animal model. However, unlike humans, mice are not natural hosts for S. aureus. Conversely, rabbits (the second most frequently used animal species) usually experience natural infections by $S$. aureus. In fact, numerous strains have been reported in commercial rabbitries ${ }^{12}$ ever since a sporadic mutation favored the human-to-rabbit host jump 40 years ago. ${ }^{13}$ It has been described that human skin morphologic ${ }^{14,15}$ and immunologic ${ }^{16}$ characteristics are more similar to those of rabbit than to those of mice. For this reason, the skin rabbit model is more appropriate than the murine one if the objective is to simulate $S$. aureus infection in humans.

To develop experimental infections using human $S$. aureus strains in mice, and even in rabbits, ${ }^{17}$ the inoculation of an extraordinary large number of bacteria is necessary as they are strains that have not adapted to these hosts, which places at a distance the results obtained in the laboratory from what actually occurs under natural conditions. So in these cases, animal models can be unreliable predictors of either the potential success of therapeutic or preventive interventions or the roles played by specific determinants of bacterial virulence in infection. ${ }^{2}$ This is why it is necessary to develop better animal models of colonization that are more representative of what happens in in vivo situations. ${ }^{3}$

This study presents, for the first time, a detailed description of staphylococcal dermal infection in a rabbit model at low doses to mimic natural infection and to approach what should occur in natural infections in humans in the most realistic way possible. The specific objectives of this study are to: describe and characterize a low-dose rabbit $S$. aureus intradermal infection model using a wild-type reference strain and several mutant strains in different key pathogenicity factors that somehow contribute in abscess formation; confirm the utility of this model after infection with a human adapted-to-rabbit (rabbitized) S. aureus strain; and compare their response with different rabbit strains of known virulence.

\section{Materials and Methods}

\section{General Approach}

This study was designed in two phases: the first intended to (1a) establish a detailed description of the evolution of lesions and the immune response after infection with low doses of a wildtype $S$. aureus rabbit strain and (1b) study the effect of different genes on the pathogenesis of intradermal staphylococcal infections. The second intended to (2) prove the reproducibility of this experimental model with a human rabbitized strain.

\section{Bacterial Strains and Growth Conditions}

The $S$. aureus strains used in this study were: Jwt (a rabbit ST121 wild-type strain isolated from a natural case of staphylococcosis) and deletion strains isogenic coa/ $w$ w
$(\mathrm{J} \Delta \operatorname{coa} \Delta v w b), \quad h l a(\mathrm{~J} \Delta h l a), \quad p s m \alpha(\mathrm{J} \Delta p s m \alpha)$, and $a g r$ $(\mathrm{J} \Delta$ agr $)$; $\mathrm{Jrot}^{+}$(Jwt with rot gen restored); $\mathrm{J} d l t \mathrm{~B}^{\mathrm{h}}$ (Jwt with the reversion of three identified $d l t \mathrm{~B}$ single-nucleotide polymorphisms); and $\mathrm{Fdlt}_{\mathrm{B}} \mathrm{r}^{\mathrm{r}}$, a human ST121 strain expressing $d l t \mathrm{~B}$ from rabbit clones ${ }^{13}$ (Table 1 ).

Bacteria were grown at $37^{\circ} \mathrm{C}$ overnight on trypticase soy agar medium supplemented with antibiotics as appropriate. Broth cultures were grown at $37^{\circ} \mathrm{C}$ in trypticase soy broth with shaking (240 revolutions per minute). The procedures for the preparation and analysis of phage lysates and transduction and transformation in $S$. aureus were performed essentially as previously described. ${ }^{20,21}$

\section{DNA Methods}

General DNA manipulations were performed by following standard procedures. ${ }^{22}$

To produce the mutant strains, plasmid pMAD was used, ${ }^{23}$ as previously described. ${ }^{24}$ The oligonucleotides used herein are listed in Table 2. Briefly, two separate PCR products with overlapping sequences, including the targeted sequence, were combined. A second PCR was run with external primers to obtain a single fragment. Specifically, 1 $\mathrm{mL}$ of each of the first PCRs was mixed with $10 \mathrm{pmol} / \mathrm{L}$ of the outside primers and was PCR amplified. The fusion products were purified and cloned at the appropriate sites of shuttle plasmid pMAD, and the resulting plasmids were transformed into $S$. aureus (RN4220) by electroporation. ${ }^{20}$ pMAD contains a temperature-sensitive origin of replication and an erythromycin-resistance gene. The plasmid was integrated into the chromosome through homologous recombination at a nonpermissive temperature $\left(43.5^{\circ} \mathrm{C}\right)$. From the $43.5^{\circ} \mathrm{C}$ plate, one to five colonies were picked into $10 \mathrm{~mL}$ of trypticase soy broth and incubated for 24 hours at $30^{\circ} \mathrm{C}$. Ten-fold serial dilutions of this culture in sterile trypticase soy broth were plated on trypticase soy agar containing X-gal (5-bromo-4-chloro-3-indolyl-B-D-galactopyranoside; $150 \mathrm{mg} / \mathrm{mL}$ ). White colonies, which no longer contained the pMAD plasmid, were tested to confirm replacement by DNA sequencing. Primers were obtained from Invitrogen Life Technologies (Paisley, UK).

\section{Animals and Sampling Times}

A total of 220 2-month-old albino hybrid rabbits (Oryctolagus cuniculus; 10 animals per sampling time) of either sex were inoculated with the eight above-described $S$. aureus strains. Depending on the inoculated strain, samples were taken at different times (Table 1). After a detailed study of lesions induced by the Jwt strain on different days postinfection (dpi; 0 , $0.5,1,2,3,7,14,21$, and 28), it was established that 7 dpi was the optimal time to compare infections with mutants in various toxins ( $\mathrm{J} \Delta \operatorname{coa} \Delta v w b, \mathrm{~J} \Delta h l a$, and $\mathrm{J} \Delta p \operatorname{sm} \alpha$ ) and general regulators ( $\mathrm{J} \Delta a g r)$ as it was the time of maximum lesion development (abscesses opening and emptying) and when repair phenomena commenced. Then, 1, 3, and 7 dpi were selected to compare the 
Table 1 Number of Rabbits Inoculated with Different Staphylococcus aureus Strains, Sampling Days, Expected Outcomes after Infection, and the Study Where They Were Involved

\begin{tabular}{|c|c|c|c|c|}
\hline Strain & Rabbits, $n$ & Sampling days & Objective & Study \\
\hline Jwt & 90 & $0,0.5,1,2,3,7,14,21,28$ & $\begin{array}{l}\text { To describe the model in detail after the intradermal inoculation } \\
\text { of a virulent } S \text {. aureus strain of rabbit origin. }\end{array}$ & $1 \mathrm{a}$ \\
\hline $\mathrm{J} \Delta \operatorname{coa} \Delta v w b$ & 10 & 7 & $\begin{array}{l}\text { Both coagulases Coa and } v W b p \text { are required for abscesses to form. } \\
\text { A reduction in the virulence of } \mathrm{J} \Delta \text { coa } \Delta v w b \text { and the presence of } \\
\text { differences in the morphology of abscesses were expected. }\end{array}$ & $1 b$ \\
\hline $\mathrm{J} \Delta h \mathrm{hla}$ & 10 & 7 & $\begin{array}{l}\alpha \text {-Hemolysin is a cytolitic toxin that promotes dermonecrosis in } \\
\text { animal skin infections. }{ }^{1} \text { A reduction in abscess size and fewer } \\
\text { and milder dermonecrotic lesions were expected. }\end{array}$ & $1 b$ \\
\hline $\mathrm{J} \Delta p s m \alpha$ & 10 & 7 & $\begin{array}{l}\text { PSM } \alpha \text { performs cytolytic (lysis of erythrocytes and neutrophils) } \\
\text { and immunomodulation (induces tolerogenic phenotypes in } \\
\text { dendritic cells and inhibits type } 1 \text { helper T-cell differentiation) } \\
\text { activities. }{ }^{3} \text { Diminished reduction in the severity of dermal } \\
\text { lesions was expected. }\end{array}$ & $1 b$ \\
\hline $\mathrm{J} \Delta \mathrm{agr}$ & 10 & 7 & $\begin{array}{l}\text { Agr regulates most } S \text {. aureus toxins and exoenzymes. }{ }^{18} \mathrm{~A} \\
\text { dysfunctional strain in this global virulence regulator should } \\
\text { reduce the high toxicity in strongly aggressive } S \text {. aureus strains. }\end{array}$ & $1 b$ \\
\hline $\mathrm{Fdlt} \mathrm{B}^{\mathrm{r}}$ & 30 & $1,3,7$ & $\begin{array}{l}\mathrm{F} \text { is a human ST121 strain that does not infect rabbits. }{ }^{13} \text { After its } \\
\text { rabbitization (introduction of most } 5^{\prime} \text { nonsynonymous SNPs at } \\
\text { the } d l t \mathrm{~B}^{*} \text { locus from rabbit strains), the virulence of Fdlt } \mathrm{B}^{\mathrm{r}} \text { was } \\
\text { expected to be similar to that of Jwt. }\end{array}$ & 2 \\
\hline $\operatorname{Jrot}^{+}$ & 30 & $1,3,7$ & $\begin{array}{l}\text { Rot is a global virulence regulator, a repressor of toxins, }{ }^{5} \text { which is } \\
\text { altered in rabbits. Its restoral is expected to make Jrot }{ }^{+} \text {less } \\
\text { virulent than Jwt. }\end{array}$ & 2 \\
\hline$J d l t \mathrm{~B}^{\mathrm{h}}$ & 30 & $1,3,7$ & $\begin{array}{l}\text { After the reversion of the three identified } d l t B \text { SNPs }\left(d l t B^{h}\right) \text { in a } \\
\text { rabbit strain } J(J w t) \text {, loss of infectivity was expected. }{ }^{13}\end{array}$ & 2 \\
\hline
\end{tabular}

${ }^{*}$ The $d l t B$ gene is involved in the resistance of $S$. aureus to positively charged antimicrobial peptides, such as defensins and other host defense peptides. ${ }^{19}$ Agr, accessory gene regulator; PSM $\alpha$, phenol-soluble modulin $\alpha$; SNP, single-nucleotide polymorphism.

behavior of a human rabbitized strain $\left(\mathrm{F} d l t \mathrm{~B}^{\mathrm{r}}\right)$ with three rabbit strains ( $\mathrm{Jwt}, \mathrm{Jrot}{ }^{+}$, and $\mathrm{J} d l t \mathrm{~B}^{\mathrm{h}}$ ) of well-known virulence in the acute and early chronic phases of infection evolution because, as before ( $7 \mathrm{dpi}$ ), some important milestones were observed: on $1 \mathrm{dpi}$, externally evident lesions (papules) and the formation of initial abscesses; and on 3 dpi, skin necrosis, multiple Splendore-Hoeppli phenomena, and an eosinophilic layer surrounding abscesses.

Animals were housed under conventional environmental conditions with an alternating cycle of 16 hours of light and 8 hours of darkness in individual cages $(600 \times 750 \times 600$ $\mathrm{mm}$ ) and were fed with a commercial rabbit diet ad libitum.

The experimental protocol was approved by the Ethical Committee of the Universidad CEU Cardenal Herrera and by the Conselleria d'Agricultura, Pesca i Alimentacio, Generalitat Valenciana (permit numbers 2011/010 and 2017/VSC/PEA/ 00192; date of approval, January 20, 2011). All the animals were handled according to the principles of animal care published by Spanish Royal Decree 1201/2005 (Boletín Oficial del Estado, 2005).

\section{Intradermal Infection Model}

The experimental procedure was performed as previously described, ${ }^{25}$ with some modifications. Briefly, rabbits were intradermally inoculated in their backs with 300 colony-forming units (CFUs) of each studied strain suspended in $0.1 \mathrm{~mL}$ of phosphate-buffered saline to inoculate animals with the lowest infective doses of bacteria. The optimal number of bacteria in the inoculum was empirically determined in the preliminary experiments. Previously, animals were sedated with a combination of ketamine (Imalgene; $100 \mathrm{mg} / \mathrm{mL}$; Merial, Barcelona, Spain) and xylazine (Xilagesic; $200 \mathrm{mg} / \mathrm{mL}$; Calier, Barcelona, Spain) and a $10 \times 10-\mathrm{cm}$ area of the dorsal-lumbar region was shaved and disinfected with chlorhexidine. To avoid interactions among strains, each rabbit was infected with only one strain (except in the phase 1b, where Jwt was inoculated as control, which were inoculated in duplicate). The general status, weight, and rectal temperature of animals were recorded daily.

\section{Gross, Microscopic, and Microbiological Studies}

The characteristics of the skin gross lesions (presence of erythema, edema, skin elevation, nodules, dermonecrosis, and/or ulceration) were recorded daily. Abscess dimensions were measured with a caliper. The length (L) and width (W) values were used to calculate abscess areas $(\mathrm{A}=\pi[\mathrm{L} \times$ $\mathrm{W}] / 2)$ and volumes $\left(\mathrm{V}=4 / 3 \pi[\mathrm{L} / 2]^{2} \times[\mathrm{W} / 2]\right) .^{26,27}$ Macroscopic lesions were evaluated by their abscess area as follows: healthy (no apparent lesions), mild $\left(<0.5 \mathrm{~cm}^{2}\right)$, 
Table 2 The Oligonucleotides Used in This Study

\begin{tabular}{|c|c|c|}
\hline Oligonucleotide & Description & Sequence \\
\hline Coa-1m & $\begin{array}{l}\text { Checking the coa mutants in Jwt and Fdlt } \mathrm{B}^{r} \text { strains by } \\
\text { inside-inside PCR. }\end{array}$ & F: 5'-ATAGAGATGCTGGTACAGG-3' \\
\hline Coa-2c & $\begin{array}{l}\text { Checking the coa mutants in Jwt and FdltB }{ }^{r} \text { strains by } \\
\text { inside-inside PCR. }\end{array}$ & R: 5'-GCTTCCGATTGTTCGATGC-3' \\
\hline Coa-5mB & $\begin{array}{l}\text { Deletion of coa }(\Delta c o a) \text { in Jwt and } F d l t B^{r} \text { strains; } 5^{\prime} \\
\text { flanking region. }\end{array}$ & F: 5'-cgcggatccTCATGACGATACTTTCAGAGG-3' \\
\hline Coa-6c & $\begin{array}{l}\text { Deletion of coa }(\Delta c o a) \text { in Jwt and FdltB }{ }^{r} \text { strains; } 5^{\prime} \\
\text { flanking region. }\end{array}$ & R: 5'-CCCAATCTACATTAAAGAAAC-3' \\
\hline Coa-7m & $\begin{array}{l}\text { Deletion of coa }(\Delta c o a) \text { in Jwt and FdltB' strains; } 3^{\prime} \\
\text { flanking region. }\end{array}$ & $\begin{array}{l}\text { F: } 5^{\prime} \text {-GGTAATTACATTTTGGAGGAAAACT } \\
\text { CTATCCATAGACATACAG-3' }\end{array}$ \\
\hline Coa-8cE & $\begin{array}{l}\text { Deletion of coa }(\Delta c o a) \text { in Jwt and Fdlt } \mathrm{B}^{\mathrm{r}} \text { strains; } 3^{\prime} \\
\text { flanking region. }\end{array}$ & R: 5'-ccggaattcGTACGAATGTGAATGGTGGC-3' \\
\hline Coa-11m & $\begin{array}{l}\text { Checking the coa mutants in Jwt and FdltB }{ }^{r} \text { strains by } \\
\text { outside-outside PCR. }\end{array}$ & F: 5'-CATACTTCGATCGTTCTATAAG-3' \\
\hline Coa-12c & $\begin{array}{l}\text { Checking the coa mutants in Jwt and FdltB' strains by } \\
\text { outside-outside PCR. }\end{array}$ & R: 5'-TTGCATCTATTAAAGAAGTAGG-3' \\
\hline$P S M \alpha-1 m B$ & $\begin{array}{l}\text { Deletion of } p s m \alpha \text { locus }(\Delta p s m \alpha) \text { in Jwt and } \mathrm{FdltB}^{\mathrm{r}} \\
\text { strains; } 5^{\prime} \text { flanking region. }\end{array}$ & F: 5'-cgggatcCACATGTTGACCATGAATACC-3' \\
\hline$P S M \alpha-2 c$ & $\begin{array}{l}\text { Deletion of psm } \alpha \text { locus }(\Delta p s m \alpha) \text { in Jwt and } \mathrm{Fdlt}^{\mathrm{r}} \\
\text { strains; } 5^{\prime} \text { flanking region. }\end{array}$ & $\begin{array}{l}\text { R: 5'-gtattcaattcgcttaaattattCATTAAG } \\
\text { АTTACСТCСTTTGC-3' }\end{array}$ \\
\hline PSM $\alpha-3 m$ & $\begin{array}{l}\text { Deletion of } p s m \alpha \text { locus }(\Delta p s m \alpha) \text { in Jwt and } \mathrm{Fdlt}^{\mathrm{r}}{ }^{\mathrm{r}} \\
\text { strains; } 3^{\prime} \text { flanking region. }\end{array}$ & F: 5'-AATAATTTAAGCGAATTGAATAC-3' \\
\hline$P S M \alpha-4 c E$ & $\begin{array}{l}\text { Deletion of psm } \alpha \text { locus }(\Delta p s m \alpha) \text { in Jwt and } F d l t B^{r} \\
\text { strains; } 3^{\prime} \text { flanking region. }\end{array}$ & R: 5'-ccggaattcAATTGTGGCTTAATTGTTTGC-3' \\
\hline$P S M \alpha-5 m$ & $\begin{array}{l}\text { Checking the psm } \alpha \text { locus mutants in Jwt and Fdlt } \mathrm{B}^{r} \\
\text { strains by inside-inside PCR. }\end{array}$ & F: 5'-GCAAAGGAGGTAATCTTAATG-3' \\
\hline$P S M \alpha-8 c$ & $\begin{array}{l}\text { Checking the psm } \alpha \text { locus mutants in Jwt and Fdlt } \mathrm{B}^{r} \\
\text { strains by inside-inside PCR. }\end{array}$ & R: 5'-CGAATTCCATGTGAATGGC- $3^{\prime}$ \\
\hline $\operatorname{PSM} \alpha-6 m$ & $\begin{array}{l}\text { Checking the psm } \alpha \text { locus mutants in Jwt and Fdlt } \mathrm{B}^{r} \\
\text { strains by outside-outside PCR. }\end{array}$ & F: 5'-TACAAAGCCAGCTAATAACC-3' \\
\hline$P S M \alpha-7 c$ & $\begin{array}{l}\text { Checking the } p s m \alpha \text { locus mutants in Jwt and Fdlt } \mathrm{B}^{r} \\
\text { strains by outside-outside PCR. }\end{array}$ & R: 5'-AATGGCAAATTAGACCAGC- $3^{\prime}$ \\
\hline vwb-1mB & $\begin{array}{l}\text { Deletion of } v w b(\Delta v w b) \text { in Jwt and } \mathrm{Fdlt}^{\mathrm{r}} \mathrm{B}^{\mathrm{s}} \text { strains; } 5^{\prime} \\
\text { flanking region. }\end{array}$ & F: 5'-cgcggatccTCAGGTTCTAACAATAATGTAG-3 \\
\hline vwb-2c & $\begin{array}{l}\text { Deletion of } v w b(\Delta v w b) \text { in Jwt and } \mathrm{Fdlt}^{\mathrm{r}} \mathrm{B}^{\mathrm{s}} \text { strains; } 5^{\prime} \\
\text { flanking region. }\end{array}$ & R: 5'-GCTCCCAATGATAAAACTAGC-3' \\
\hline vwb-3m & $\begin{array}{l}\text { Deletion of } v w b(\Delta v w b) \text { in Jwt and } \mathrm{Fdlt}^{\mathrm{r}} \mathrm{B}^{\mathrm{s}} \text { strains; } 3^{\prime} \\
\text { flanking region. }\end{array}$ & $\begin{array}{l}\text { F: 5'-gctagttttatcattgggagcAAGCAA } \\
\text { ATAATGAGTTTGCCG-3' }\end{array}$ \\
\hline$v w b-4 c E$ & $\begin{array}{l}\text { Deletion of } v w b(\Delta v w b) \text { in Jwt and } \mathrm{FdltB}^{\mathrm{r}} \text { strains; } 3^{\prime} \\
\text { flanking region. }\end{array}$ & R: 5'-cCggaattcTTTGTTGTCAGCTAAACTTCC-3' \\
\hline vwb-7m & $\begin{array}{l}\text { Checking the } v w b \text { mutants in Jwt and Fdlt } \mathrm{B}^{r} \text { strains by } \\
\text { outside-outside PCR; } 3^{\prime} \text { flanking region. }\end{array}$ & F: 5'-ACCAATTCCAGAGGATTCAG-3' \\
\hline vwb-11m & $\begin{array}{l}\text { Checking the } v w b \text { mutants in Jwt and Fdlt } \mathrm{B}^{r} \text { strains by } \\
\text { outside-outside PCR; } 3^{\prime} \text { flanking region. }\end{array}$ & F: 5'-GGCTGAAGATGAGGCTTTG-3' \\
\hline vwb-8c & $\begin{array}{l}\text { Checking the } v w b \text { mutants in Jwt and Fdlt } \mathrm{B}^{r} \text { strains by } \\
\text { outside-outside PCR; } 5^{\prime} \text { flanking region. }\end{array}$ & R: 5'-TATTCAATTTATCTTCAGAAGC-3' \\
\hline vwb-12c & $\begin{array}{l}\text { Checking the } v w b \text { mutants in Jwt and Fdlt } \mathrm{B}^{r} \text { strains by } \\
\text { outside-outside PCR; } 5^{\prime} \text { flanking region. }\end{array}$ & R: 5'-TAAGTCGCACTTTAATTGC-3' \\
\hline
\end{tabular}

The sequences recognized by the restriction enzymes used in cloning are denoted by lowercase letters.

$F$, forward; $R$, reverse.

moderate $\left(0.5\right.$ to $\left.5 \mathrm{~cm}^{2}\right)$, or severe $\left(>0.5 \mathrm{~cm}^{2}\right.$ and gross necrosis).

On sampling, rabbits were sedated with a combination of ketamine (Imalgene; $100 \mathrm{mg} / \mathrm{mL}$ ) and xylazine (Xilagesic; $200 \mathrm{mg} / \mathrm{mL}$ ), and were euthanized by an i.v. injection of barbiturate (T-61; Intervet International $\mathrm{GmbH}$, Unterschleißheim, Germany); a complete necropsy was performed. Skin samples from the inoculation place were used for the microscopic and microbiological studies and the cytokine assay. For the histologic examination, skin samples were 
Table 3 The Monoclonal Antibodies Used in the Immunohistochemical Studies

\begin{tabular}{|c|c|c|c|c|c|c|}
\hline $\begin{array}{l}\text { Primary antibodies } \\
\text { (dilution) }\end{array}$ & Isotype & Reactivity & Antigen retrieval & $\begin{array}{l}\text { Reference } \\
\text { (manufacturer) }\end{array}$ & $\begin{array}{l}\text { Secondary } \\
\text { antibodies } \\
\text { (dilution) }\end{array}$ & $\begin{array}{l}\text { Reference } \\
\text { (manufacturer) }\end{array}$ \\
\hline $\begin{array}{l}\text { Mouse anti-rabbit } \\
\text { macrophage } \\
\text { clone RAM11 } \\
(1: 50)\end{array}$ & IgG1k & Macrophages & $\begin{array}{l}\text { Enzymatic } \\
\text { treatment }\end{array}$ & $\begin{array}{l}\text { M0633 (Dako, } \\
\text { Capertina, CA) }\end{array}$ & $\begin{array}{l}\text { Biotinylated anti- } \\
\text { mouse IgG } \\
\text { ( } 1 \text { drop into } \\
10 \mathrm{~mL} \text { of diluent) }\end{array}$ & $\begin{array}{c}\text { PK } 6102 \text { (Vector } \\
\text { Laboratories) }\end{array}$ \\
\hline
\end{tabular}

HRP, horseradish peroxidase.

routinely stained with hematoxylin and eosin, Masson trichrome, and Gram's stains, and were processed by immunohistochemistry for macrophages, $\mathrm{T}$ and $\mathrm{B}$ lymphocytes, and plasma cell detection by the avidin-biotin-peroxidase complex method at the dilutions recommended by the manufacturer (Table 3). In the immunohistochemical procedure, positive cells were enumerated in the inoculation areas and, when lesions were severe, in the border of the necrotized tissue of 20 $0.08-\mathrm{mm}^{2}$ randomly selected fields per slide. The histologic and immunohistochemical findings observed on the different skin layers were described and recorded in detail.

Before fixation, representative samples were taken from skin lesions and kidneys, and were weighed under sterile conditions for the microbiological studies. Then, they were immersed in a cold solution of phosphate-buffered saline and mechanically homogenized in the presence of ice. Tissue homogenate $(100 \mu \mathrm{L})$ was cultivated on blood-agar (BioMérieux, Marcy l'Etoile, France) and was incubated aerobically at $37^{\circ} \mathrm{C}$ for 24 hours. This allows the results to be expressed in CFUs/g. The colonies that grew were identified by coagulase $\mathrm{PCR}^{12}$ to prove that the strains isolated from skin lesions were the same as those originally inoculated and to assess the possibility of septicemia in the samples taken from kidneys.

\section{Hematology and Flow Cytometric Analysis}

Blood samples $(1 \mathrm{~mL})$ in EDTA anticoagulant were obtained from the median artery of the ear. White blood cell counts and lymphocyte proportions were determined using a

Table 4 The Monoclonal Antibodies Used in the Flow Cytometry Studies

\begin{tabular}{|c|c|c|c|c|c|}
\hline Monoclonal antibodies & Isotype & Specificity & Reactivity & Clone & Reference (manufacturer) \\
\hline Mouse anti-rabbit IgM & IgG1 & $\operatorname{IgM}$ & B cells & NRBM & $\begin{array}{l}\text { MCA812GA (AbD Serotec, } \\
\text { Kidlington, UK) }\end{array}$ \\
\hline $\begin{array}{l}\text { Mouse anti-rabbit T lymphocytes: } \\
\text { FITC* }^{*}\end{array}$ & IgG1 & CD5 & $\mathrm{T}$ cells & KEN-5 & MCA800F (AbD Serotec) \\
\hline Mouse anti-rabbit CD4 & $\operatorname{IgG} 2 a$ & CD4 & T CD4 cells & KEN-4 & MCA799 (AbD Serotec) \\
\hline Mouse anti-rabbit CD8 & $\operatorname{IgG} 2 \mathrm{a}$ & CD8 & T CD8 cells & ISC27A & $\begin{array}{l}\text { WS0796U-100 (Kingfisher } \\
\text { Biotech, St. Paul, MN) }\end{array}$ \\
\hline Mouse anti-human CD14: FITC & $\operatorname{IgG} 2 \mathrm{a}$ & CD14 & Monocytes and granulocytes & TÜK4 & MCA1568F (AbD Serotec) \\
\hline Mouse anti-rabbit $\alpha-C D 45$ & $\operatorname{IgM}$ & CD45 & All leukocytes & ISC76A & $\begin{array}{l}\text { ISC76A (Veterinary Medical } \\
\text { Research \& Development, } \\
\text { Pullman, WA) }\end{array}$ \\
\hline
\end{tabular}

*Clon KEN-5 recognizes rabbit T lymphocytes and immunoprecipitates. This antibody recognizes rabbit CD5 but does not bind to rabbit CD5 transfectants. Known rabbit CD5 antibodies also show binding to most B lymphocytes, which are not labeled by this clone (information obtained from data sheet).

FITC, fluorescein isothiocyanate. 
A

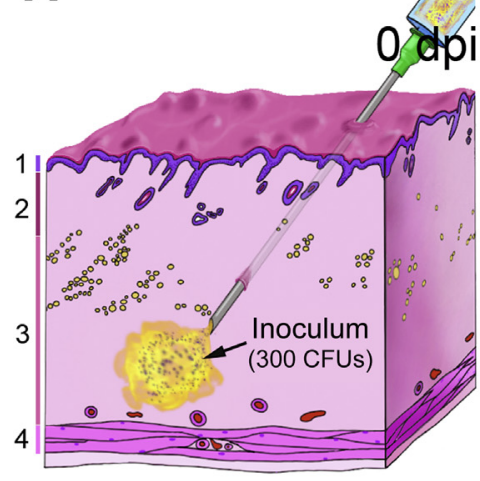

D

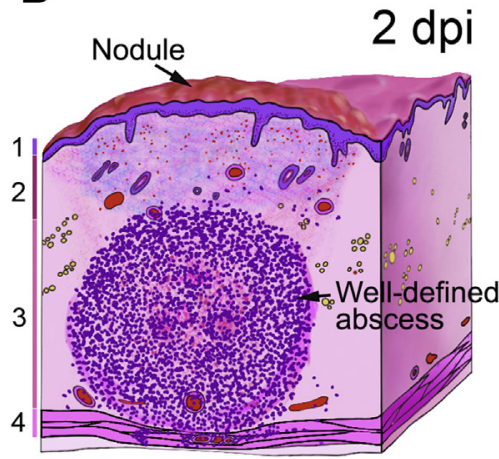

B

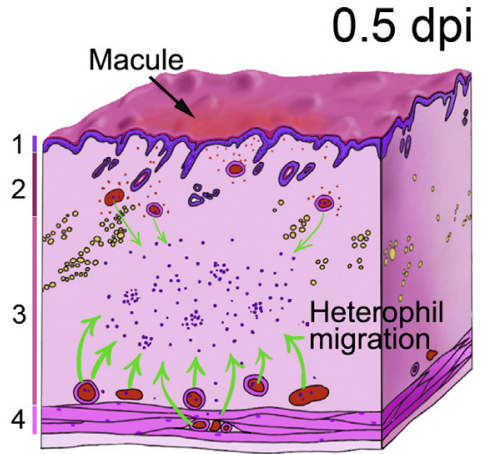

C

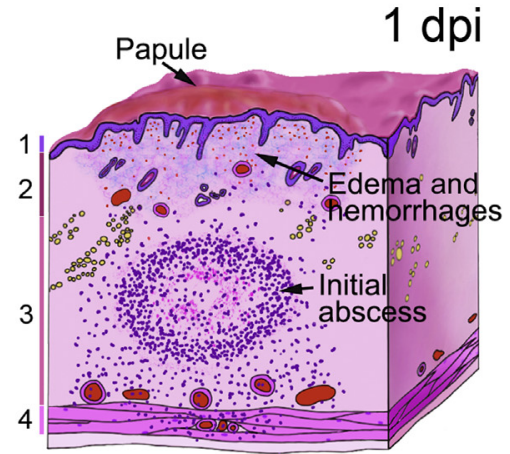

E

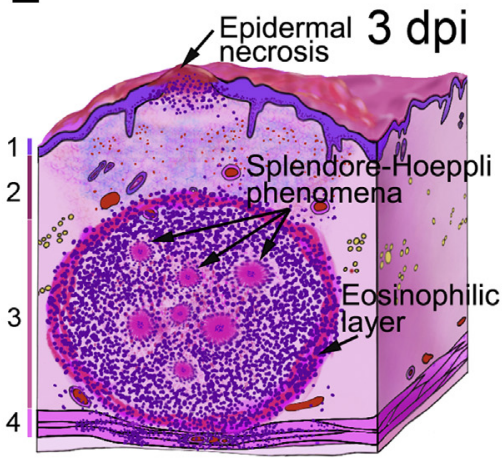

F

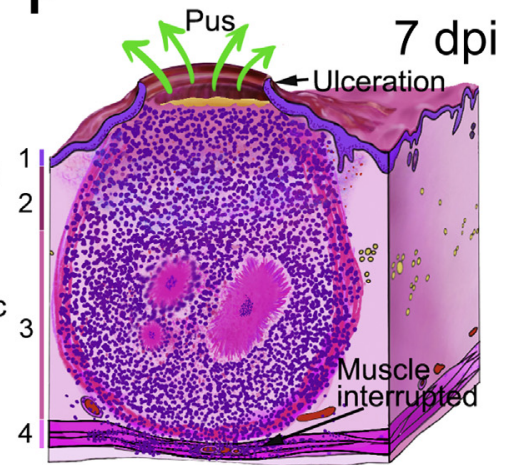

G

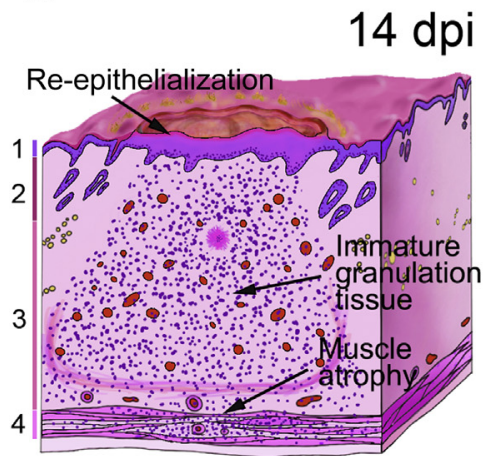

H

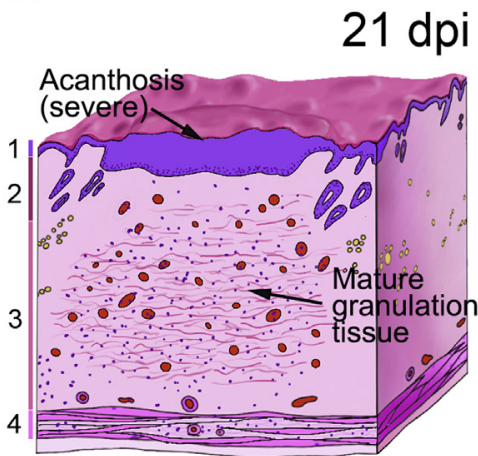

I

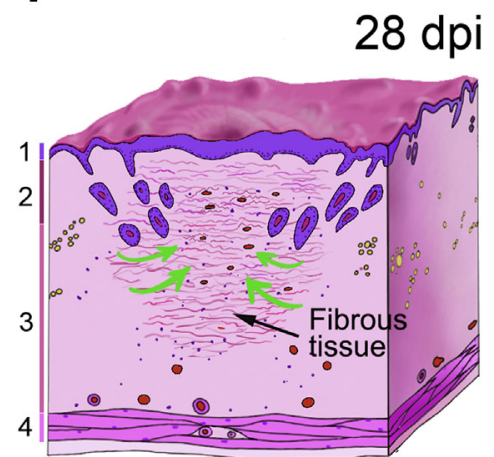

Figure 1 Representation of abscess evolution after experimental infection with Staphylococcus aureus (Jwt strain) with time. A: At 0 days postinoculation (dpi). Intradermal inoculation of 300 colony-forming units (CFUs) of S. aureus. Colored bars and numbers indicate the different histologic parts of skin (1, epidermis; 2, superficial dermis; 3, deep dermis; and 4, cutaneous muscle). B: At 0.5 dpi. 1: Externally, reddish macule. 2: Mild vascular phenomena (edema and hemorrhages). 3: Migration of heterophils mainly from deep vessels (arrows) and grouping at the inoculation point. 4: Small hemorrhages. C: At $1 \mathrm{dpi}$. 1: External slightly elevated reddish papule. 2: Mild to moderate edema and hemorrhages. 3: Initial abscess structured as a center of eosinophilic necrosis, with some bacteria surrounded by diffusely distributed heterophils. 4: Muscular cells moderately infiltrated by heterophils. D: At 2 dpi. 1: External reddish and elevated nodule and mild epidermal acanthosis. 2: Moderate edema and hemorrhages. 3: Well-defined abscess composed of a center of eosinophilic necrosis with bacteria, surrounded by a dense layer of heterophils. 4: Muscle cells infiltrated by numerous heterophils that induce their separation and necrosis. E: At 3 dpi. 1: Big dark nodule with epidermal necrosis and acanthosis. 2: Severe vascular phenomena. 3: Abscess with the presence of multiple Splendore-Hoeppli phenomena and an external layer of eosinophilic necrosis. 4: Myocytes severely infiltrated by heterophils, causing their degeneration. F: At 7 dpi. 1: Necrosis and skin ulceration, allowing the outflow of purulent content and acanthosis in the periphery to the ulcer. 2: Severe vascular phenomena. 3: Splendore-Hoeppli phenomena merging with one another and the presence of a thin eosinophilic layer of necrosis surrounding the entire abscess. 4: Cutaneous muscle interrupted by the presence of numerous heterophils and round cells. G: At $14 \mathrm{dpi}$. 1: External lack of hair (alopecia), ulcer re-epithelialization, and moderate to severe acanthosis. 2: Absence of skin adnexa and moderate vascular phenomena. 3: Presence of immature granulation tissue (numerous small neovessels and abundant connective tissue) and some scattered Splendore-Hoeppli phenomena. 4: Atrophy of myocytes. H: At 21 dpi. 1 : External alopecia, severe acanthosis. 2 and 3: Abundant mature granulation tissue. 4: Interstitial fibrosis and atrophy of myocytes. I: At 28 dpi. 1: External small area of alopecia and moderate acanthosis. 2: Invasion of skin appendages to the periphery of the scar. 3: Mature granulation tissue with abundant fibrous tissue. 4: Completely regenerated cutaneous muscle. 


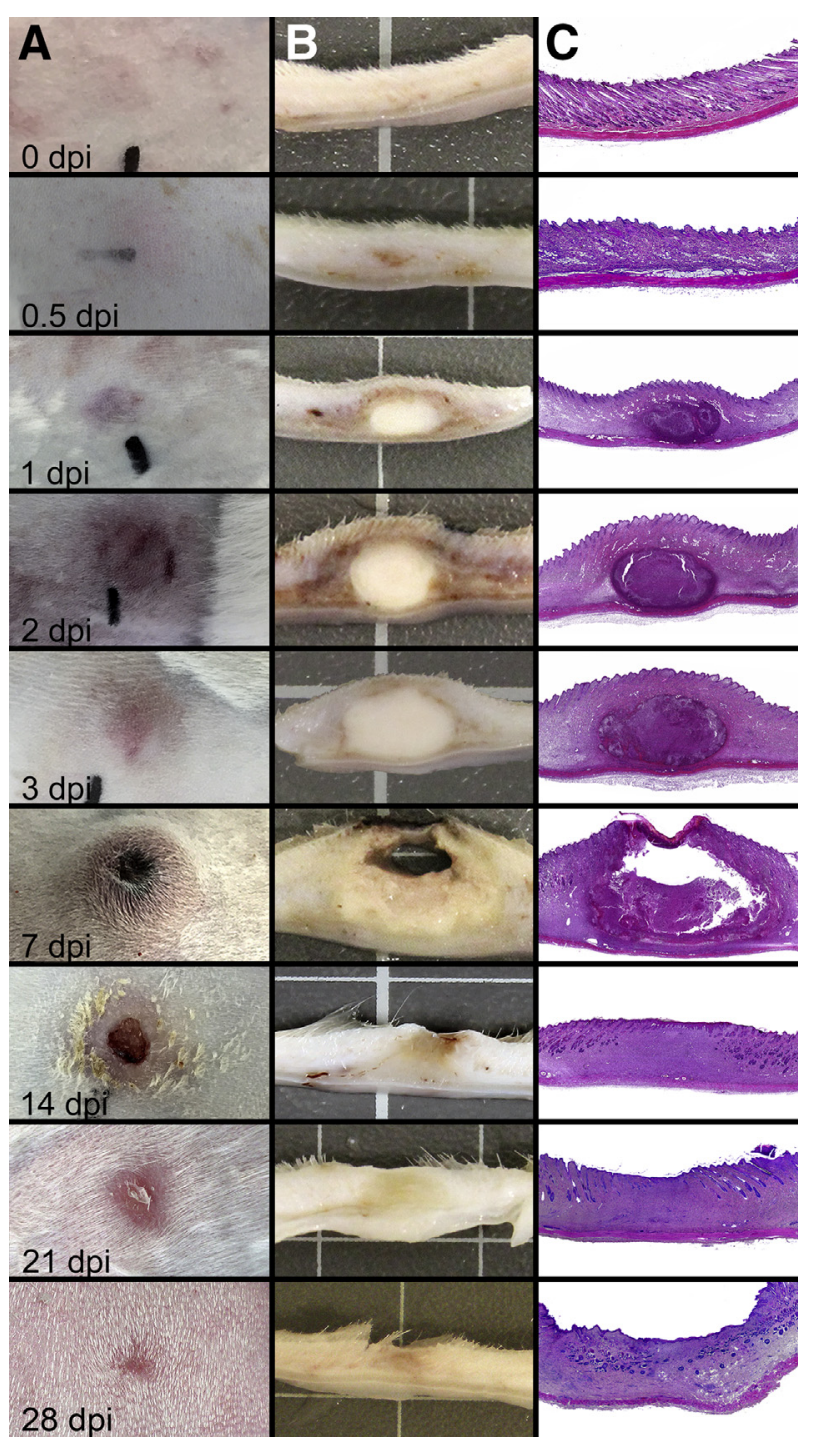

Figure 2 Correspondence between the gross (external and internally) and histologic lesions during the experiment of the wild-type (Jwt) Staphylococcus aureus strain inoculation. A: External aspect. B: Sagittal cut of formalin-fixed tissue. C: Microscopically low-magnification view stained with hematoxylin-eosin. Dpi, days postinfection.

hematology analyzer (MEK-6410; Nihon Kohden, Tokyo, Japan). The flow cytometric analysis of the white blood cells (B, T, T CD4 ${ }^{+}, \mathrm{T} \mathrm{CD}^{+}$and activated $\mathrm{T}$ cells, monocytes, and granulocytes) was performed using specific primary antibodies (Table 4) and secondary antibodies [rat antimouse $\operatorname{IgG} 2 \mathrm{a}+\mathrm{b}$ phycoerythrin conjugate (Veterinary Medical Research \& Development, Pullman, WA) and goat anti-mouse IgM phycoerythrin conjugate (AbD Serotec, Kidlington, UK)], as previously described. ${ }^{28,29}$

The common leukocyte antigen CD14 and CD45 expression was used for the lymphogate setup, as previously described. ${ }^{28,29}$ The calculation of the total lymphocyte and the respective subset counts were performed as the product of the white blood cell count and specific population percentages, as described elsewhere. ${ }^{29,30}$

\section{Cytokine Assay}

The representative ILs of the immune response [IL-1 $\beta$, IL- 4 , IL-17, IL-18, and interferon (IFN)- $\gamma$ ] from plasma and the inoculated skin tissues were analyzed by enzyme-linked immunosorbent assay kits (CUSABIO, Wuhan, China), according to the manufacturer's protocol. The samples taken from skin abscesses were immersed in a cold solution of phosphate-buffered saline with a protease inhibitor (Complete Mini; Roche, Basel, Switzerland), and were mechanically homogenized for 1 minute by stopping each 10 seconds to avoid overheating in a T50 Ultra-Turrax (IKA, Staufen, Germany) in the presence of ice. Next, they were centrifuged at $13,000 \times g$ for 10 minutes. Aliquots of the supernatant were stored at $-80^{\circ} \mathrm{C}$ for further processing.

\section{Statistical Analysis}

\section{Study 1a: Characterization of the Model after Infection with Jwt}

The data obtained in this study were evaluated by five different models, depending on the type of data recorded for each trait (Table 1 and Supplemental Table S1). The five models included the postinoculation day (nine levels: 0, 0.5, 1, 2, 3, 7, 14, 21, and 28 ) as the fixed effect. Except for CFUs, the data of quantitative traits with one record per animal were analyzed by a linear model M1 (proc GLM; SAS version 9.2; SAS Institute, Cary, $\mathrm{NC)}$. CFU usually presents changes in the order of magnitude as infections evolve. Considering this situation, the CFU data were analyzed by a mixed model M2 and by considering different variances for the random residual errors on each postinoculation day. The data of the quantitative traits with more than one record per animal were analyzed by a mixed model M3 (proc MIXED, SAS version 9.2), which included the effect of the individual on infection as random effects [90 levels; $\left.\mathrm{N} \sim\left(0, \sigma_{\mathrm{p}}\right)\right]$. The data of the categorical traits with two levels (eg, yes/no, presence/ absence, $0 / 1$ ) were analyzed by a generalized linear model M4 (proc GENMOD, SAS version 9.2) after considering that the response variable followed a binomial distribution and by using logistic transformation $(\ln [\mu /(1-\mu)])$ as a link function. Finally, the ordinal data of the histology traits had more than two levels because injuries were classified according to the following scale $(-,-/+,+,+/++,++,++/+++,+++$, $+++/++++,++++$ ), where ++ was greater (more severe) than + . However, this did not mean that the distance between each class was proportional. To take this situation into account, the data of the histology traits were analyzed by a generalized linear model M5 (proc GENMOD, SAS version 9.2) after considering that the response variable followed a multinomial probability distribution and that the link function followed a cumulative logistic distribution. ${ }^{31}$

Study 1b: Effects of the Selected Genes and the Global Regulator Involved in Abscess Development

A pairwise comparison of the means was performed using Yates correction. The $t$-test was performed for the traits with 


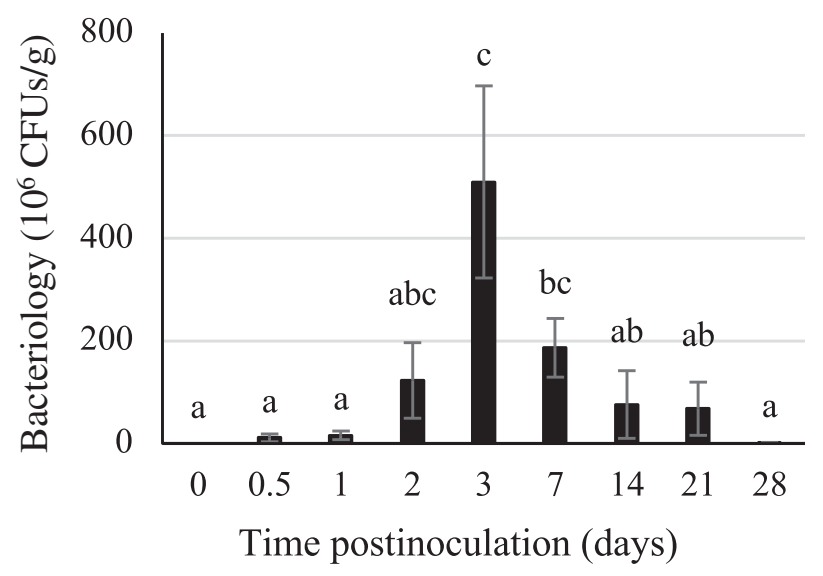

Figure 3 Colony-forming units (CFUs) after intradermal inoculation with Jwt strain of Staphylococcus aureus. Error bars correspond to the SEM for each least square mean. $n=10$ animals per time point. ${ }^{\mathrm{a}-c}$ The means not sharing superscript significantly differ $(P<0.05)$.

quantitative data, and the $\chi^{2}$ test was performed for the traits with categorical data (M6).

Study 2: Comparison of the Lesions Caused by a Human Rabbitized Strain and Different Rabbit Strains of Known Virulence

The data obtained in this study were evaluated by four different models, depending on the type of data recorded per trait (Supplemental Table S1). The four models included day postinoculation (three levels: 1, 3, and 7), the strain (four levels: $\mathrm{Jwt}, \mathrm{F} d l t \mathrm{~B}^{\mathrm{r}}$, $\mathrm{J} r o t^{+}$, and $\mathrm{J} d l t \mathrm{~B}^{\mathrm{h}}$ ), and their interaction as fixed effects. Except for CFUs, the data of the quantitative traits with only one record per animal were analyzed by a linear model M7 (proc GLM, SAS version 9.2). CFU was analyzed by a mixed model M8 after considering the different variances for the random residual errors on each day postinoculation. The data of the quantitative traits with more than one record per individual were analyzed by a mixed model M9 (proc MIXED, SAS version 9.2) and included the effect of the individual at the experimental infection time as random effects [120 levels: $\mathrm{N} \sim(0$, $\left.\sigma_{\mathrm{p}}\right)$ ]. Finally, the data of the ordinal categorical traits with more than two levels (histology variables) were analyzed by a generalized linear model M10 (proc GENMOD, SAS version 9.2) after considering that the response variable followed a multinomial probability distribution and that the link function followed a logistic distribution cumulative. ${ }^{31}$

\section{Results}

Study 1a: Characterization of the Model after Infection with a Rabbit Wild-Type Strain (Jwt)

After evidencing that rabbits can be infected by a low-dose charge of S. aureus, ${ }^{13}$ this study set out to make a detailed description, for the first time, of the pathogenesis of staphylococcal infections in the model that best mimicked staphylococcal disease in humans: the rabbit experimental model.

\section{Health Parameters}

A slight stagnation of animals' weight on the first 3 days after infection was detected, but they grew normally during the rest of the experiment. Although an increase in rectal temperature was observed during the study $\left(38.5^{\circ} \mathrm{C}\right.$ at $0 \mathrm{dpi}$ and $39.5^{\circ} \mathrm{C}$ at $28 \mathrm{dpi} ; P=0.001$ ), fever was not detected in any animal and the temperature values fell within physiological ranges.

\section{Gross Lesions}

Lesions of different severities developed in all cases after the inoculation of 300 CFUs of $S$. aureus (Figure 1A). Small $(<1 \mathrm{~cm}$ in diameter), flat, and circumscribed red macules were observed at the point of inoculation at $0.5 \mathrm{dpi}$ in $100 \%$ of the animals. These lesions evolved to slightly elevated papules ( $1 \mathrm{dpi})$ and firm nodules $(2 \mathrm{dpi})$ in $100 \%$ of the animals and at $100 \%$ of the inoculation points (20 of 20 ). The size and intensity of the reddened areas reached its apogee at $2 \mathrm{dpi}\left(2.003 \pm 0.51\right.$ versus $3.422 \pm 0.47 \mathrm{~cm}^{2}$ on 1 and 2 dpi, respectively; $P<0.001)$ and gradually disappeared after $3 \mathrm{dpi}$. At this time ( $3 \mathrm{dpi}$ ), the volume of nodules was maximum $\left(4.561 \pm 1.15 \mathrm{~cm}^{3} ; P<0.001\right)$ and $50 \%$ of the animals (5 of 10) at 7 dpi showed epidermal necrosis in the inoculation zone, characterized by darkness, and suppurative to dry (parchment) areas that opened between 7 and 14 dpi in all cases to result in ulceration and discharge of a purulent material. Draining abscesses reduced abscess size. From 14 to $28 \mathrm{dpi}$, a repair of the ulcers occurred, and absence of hair and scar retraction were observed at the end of the study (Figure 2).

\section{Bacteriology}

The bacterial counts increased at $0.5 \mathrm{dpi}\left(1.11 \times 10^{7} \mathrm{CFUs} / \mathrm{g}\right)$, with the largest number obtained at $3 \mathrm{dpi}\left(50.98 \times 10^{7} \mathrm{CFUs} / \mathrm{g}\right)$ and, coinciding with the opening of abscesses, the number of bacteria lowered until the end of the experiment, when the lesions of only 4 of the 10 animals contained bacteria $\left(1.16 \times 10^{3}\right.$ CFUs/g at $28 \mathrm{dpi}$ ) (Figure 3). All the cultured and identified bacteria recovered from lesions were the same as those inoculated, and no bacteria were isolated from blood or kidneys.

\section{Histologic Findings}

Some animals showed early histopathologic modifications at $0.5 \mathrm{dpi}$ (Table 5). In these cases, lesions were characterized by moderate edema and minor hemorrhages in the superficial dermis and by a slight increase in the density of heterophils in the deep dermis, which sometimes grouped into small clusters of cells (Figures 1B and $4 \mathrm{~A}$ ). The capillaries surrounding these inflammatory foci were hyperemic. The capillaries located near or between cutaneous muscle cells were also dilated and hyperemic, with the presence of heterophils migrating by diapedesis to the interstice. Small hemorrhagic foci 
between adipocytes near the cutaneous muscle were detected.

Vascular phenomena, such as hyperemia (vascular dilatation and presence of numerous intravascular heterophils), mild to moderate edema, and hemorrhages were detected in the superficial dermis on $1 \mathrm{dpi}$. The deep dermis showed hyperemia and numerous heterophils around dilated blood vessels and inflammatory foci structured as a core of eosinophilic necrosis, sometimes with coccoid bacteria surrounded by diffusely distributed heterophils (Figure 4B). Cutaneous muscle fibers were infiltrated by heterophils, which provoked a mild degeneration and atrophy of myocytes (Figure 1C).

Two days after infection, lesions were similar to the way they were the previous day, but presented greater severity. It was the first time that clear epidermis thickening (moderate acanthosis) was detected (10\% of animals). The presence of mild vascular dilatation, moderate edema, and some hemorrhages in the superficial dermis was observed. In the deep dermis, inflammatory foci were better well defined than they were previously. They were characterized by a center of eosinophilic necrosis, inside which there were coccoid bacteria surrounded by a dense layer of heterophils (Figure 4C). Around these foci, there were also heterophils. Cutaneous muscle cells were severely infiltrated by heterophils, which induced their separation and necrosis (Figures 1D and 4D).

On 3 dpi, the percentage of animals with moderate to severe epidermal hyperplasia was higher than on 2 dpi (46\% versus $10 \%)$. The presence of more severe vascular phenomena (vascular dilatation, edema, and hemorrhages) in the superficial dermis induced skin tumefaction, and epidermis necrosis when the volume of abscesses was sizable (Figure 4E). In the deep dermis, the severity of edemas and hyperemia diminished. The latter was observed only around suppurative foci, together with diapedesis phenomena of heterophils. Inflammatory foci were structured by a center with bacterial colonies surrounded by a star-shaped homogeneous eosinophilic material (Splendore-Hoeppli phenomenon) and an external layer of cellular debris and inflammatory cells (mainly lymphocytes, macrophages, and heterophils) (Figure 4F). When abscesses were large, this pattern was repeated, and they merged with one another to form the abscess. Externally, abscesses were partially or totally surrounded by a thin eosinophilic layer of

Table 5 The Histopathologic Findings Observed after the Inoculation of a Jwt Staphylococcus aureus Strain and Their Evolution during the Experiment

\begin{tabular}{|c|c|c|c|c|}
\hline \multirow[b]{2}{*}{ Variable } & \multicolumn{4}{|c|}{ Days postinoculation } \\
\hline & 0.5 & 1 & 2 & 3 \\
\hline \multicolumn{5}{|l|}{ Epidermis* } \\
\hline Re-epithelization (mitosis) & $100 / 0 / 0 / 0^{a}$ & $100 / 0 / 0 / 0^{a}$ & $100 / 0 / 0 / 0^{a}$ & $100 / 0 / 0 / 0^{\mathrm{a}}$ \\
\hline \multicolumn{5}{|l|}{ Superficial dermis } \\
\hline Vascular dilatation & $80 / 20 / 0 / 0^{a}$ & $30 / 60 / 10 / 0^{a b}$ & $30 / 60 / 0 / 0^{\mathrm{ab}}$ & $18 / 55 / 27 / 0^{b c}$ \\
\hline \multicolumn{5}{|l|}{ Deep dermis } \\
\hline Vascular dilatation & $80 / 20 / 0 / 0^{a}$ & $0 / 100 / 0 / 0^{\mathrm{a}}$ & $30 / 70 / 0 / 0^{a}$ & $18 / 55 / 27 / 0^{a b}$ \\
\hline Perivascular inflammation & $30 / 50 / 10 / 10^{b}$ & $0 / 20 / 10 / 70^{\mathrm{e}}$ & $10 / 10 / 30 / 50^{\text {de }}$ & $0 / 36 / 55 / 9^{c}$ \\
\hline Heterophiles diffuse & $40 / 50 / 10 / 0^{\mathrm{a}}$ & $10 / 40 / 10 / 40^{\text {bc }}$ & $10 / 20 / 50 / 20^{\mathrm{bc}}$ & $0 / 27 / 63 / 0^{b c}$ \\
\hline Heterophiles organized (abscess) & $80 / 10 / 0 / 10^{a}$ & $20 / 10 / 10 / 60^{b}$ & $20 / 10 / 10 / 60^{\text {bc }}$ & $0 / 0 / 0 / 100^{c}$ \\
\hline \multicolumn{5}{|l|}{ Cutaneous muscle } \\
\hline Interstitial inflammation & $40 / 40 / 0 / 20^{b}$ & $10 / 10 / 60 / 20^{\text {cde }}$ & $10 / 50 / 20 / 20^{b c d}$ & $0 / 28 / 36 / 36^{\text {de }}$ \\
\hline Atrophy/degeneration & $90 / 0 / 10 / 0^{a}$ & $70 / 20 / 10 / 0^{\mathrm{ab}}$ & $70 / 20 / 10 / 0^{\mathrm{ab}}$ & $35 / 55 / 10 / 0^{a b}$ \\
\hline Hypertrophy & $100 / 0 / 0 / 0$ & $100 / 0 / 0 / 0$ & $100 / 0 / 0 / 0$ & $100 / 0 / 0 / 0$ \\
\hline Interruption & $90 / 10 / 0 / 0^{a}$ & $100 / 0 / 0 / 0^{a}$ & $100 / 0 / 0 / 0^{a}$ & $70 / 20 / 10 / 0^{a b}$ \\
\hline Fibrosis & $100 / 0 / 0 / 0^{a}$ & $100 / 0 / 0 / 0^{a}$ & $100 / 0 / 0 / 0^{a}$ & $\begin{array}{c}100 / 0 / 0 / 0^{\mathrm{a}} \\
\text { (table continues) }\end{array}$ \\
\hline
\end{tabular}

For a given trait presented in a row, $P$ value tests hypothesis H0: there was no effect of treatment to explain the observed trait variance. $n=10$ animals per day.

${ }^{\mathrm{a}-\mathrm{e}}$ The means in a same row not sharing superscript significantly differ for each inoculation time $(P<0.05)$.

*Each histopathologic finding presents four values $(\mathrm{w} / \mathrm{x} / \mathrm{y} / \mathrm{z})$ that indicate the percentage of animals with microscopic findings classified as absent ( $w)$, mild $(\mathrm{x})$, moderate $(\mathrm{y})$, and severe $(\mathrm{z})$. 
necrosis (Figure 4G). The myocytes of cutaneous muscle were infiltrated by eosinophils and macrophages, and showed dilatation, degeneration, and, sometimes, atrophy (Figures 1E and $4 \mathrm{H})$.

The main characteristic on 7 dpi was the presence of local epithelial necrosis and epidermis ulceration with severe acanthosis in the peripheric epithelium (Figure 4I), together with severe hyperemia, edema, and hemorrhages in the superficial dermis. At greater depths, abscesses showed several Splendore-Hoeppli phenomena, whose peripheral inflammatory reactions merged with one another. An external thin eosinophilic layer surrounded abscesses. Around abscesses, heterophils diffusely distributed or a few were seen and some of the capillary vessels were reactive (presence of endothelial hyperplasia and hypertrophy). The cutaneous muscle was interrupted by an inflammatory infiltrate (heterophils and round cells) that caused the atrophy of the affected cells (Figure 1F).

On $14 \mathrm{dpi}$, the severity of some inflammatory phenomena started to decrease and repair appeared more evident (Figure 1G). In those cases in which the epithelium was lost, epidermis re-epithelization, characterized by the presence of numerous mitotic figures on the basal and spinosum layers at the borders of the ulcer, was observed (Figure 4J), together with the absence of skin adnexa (sweat and sebaceous glands and hair follicles). All the animals showed acanthosis. The vascular phenomena were milder, but still evident in the superficial dermis, like inflammatory foci with bacteria and, in some cases, Splendore-Hoeppli phenomena, all surrounded by an eosinophilic layer in the deep dermis. In adjacent areas, granulation tissue (numerous small vessels and abundant fibrous tissue) was observed (Figure 4K). On the muscular layer, focal fibrosis and atrophy of some cells were observed. Other locations presented hypertrophy and hyperplasia of muscular fibers.

On $21 \mathrm{dpi}$, mitotic cells and hyperplasia in the peripheral epithelium of lesions were observed, even in those few rabbits that still had ulcers in the epithelium. When lesions were completely epithelized, the epidermis was twofold to threefold thicker than in the unaffected areas (Figure 5). In the dermis (mainly in the deep dermis), abundant granulation tissue was observed, with numerous small blood vessels and heterophils forming small cellular aggregates or diffusely distributed, mainly near cutaneous muscle, which,

Table 5 (continued)

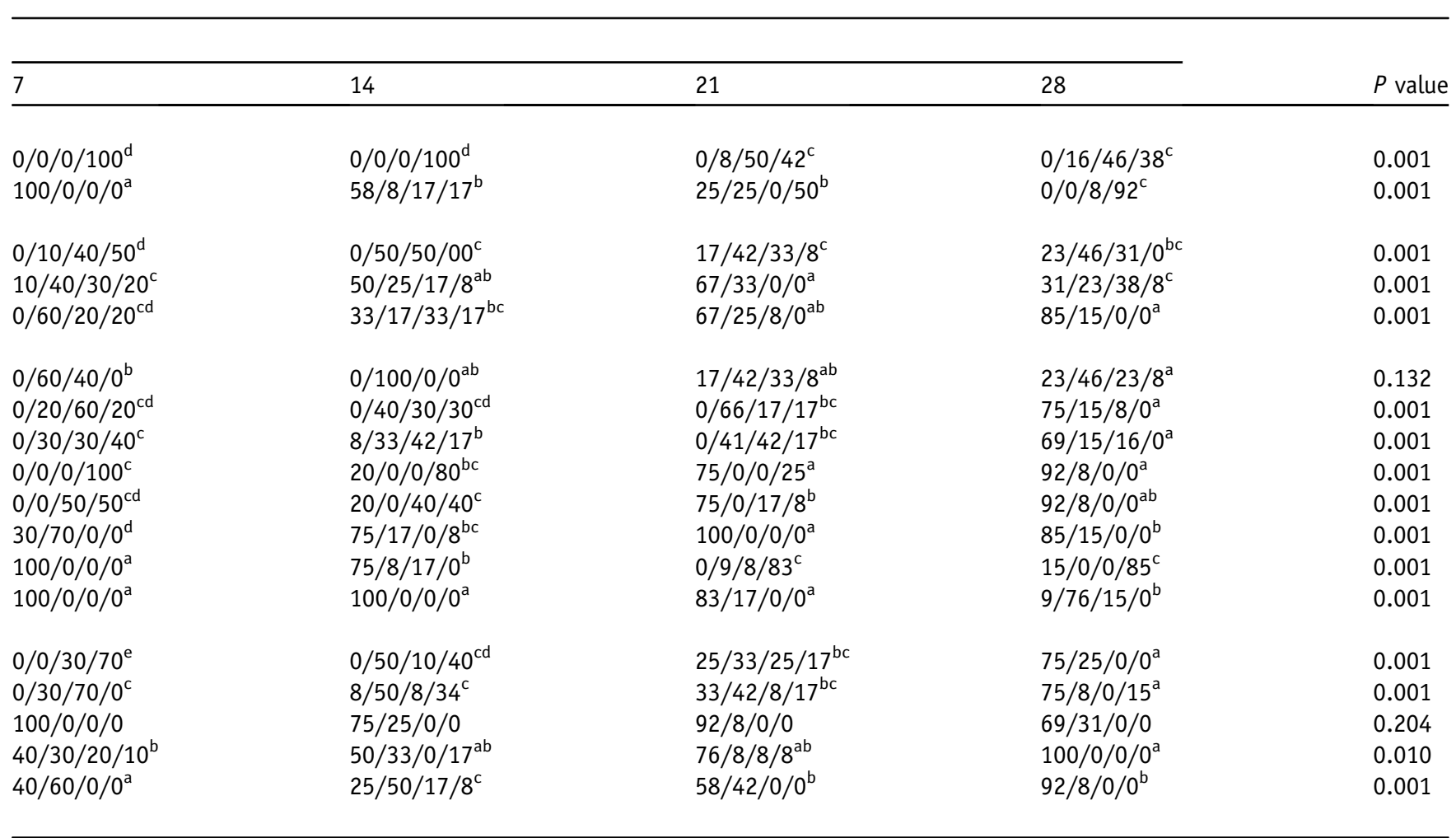




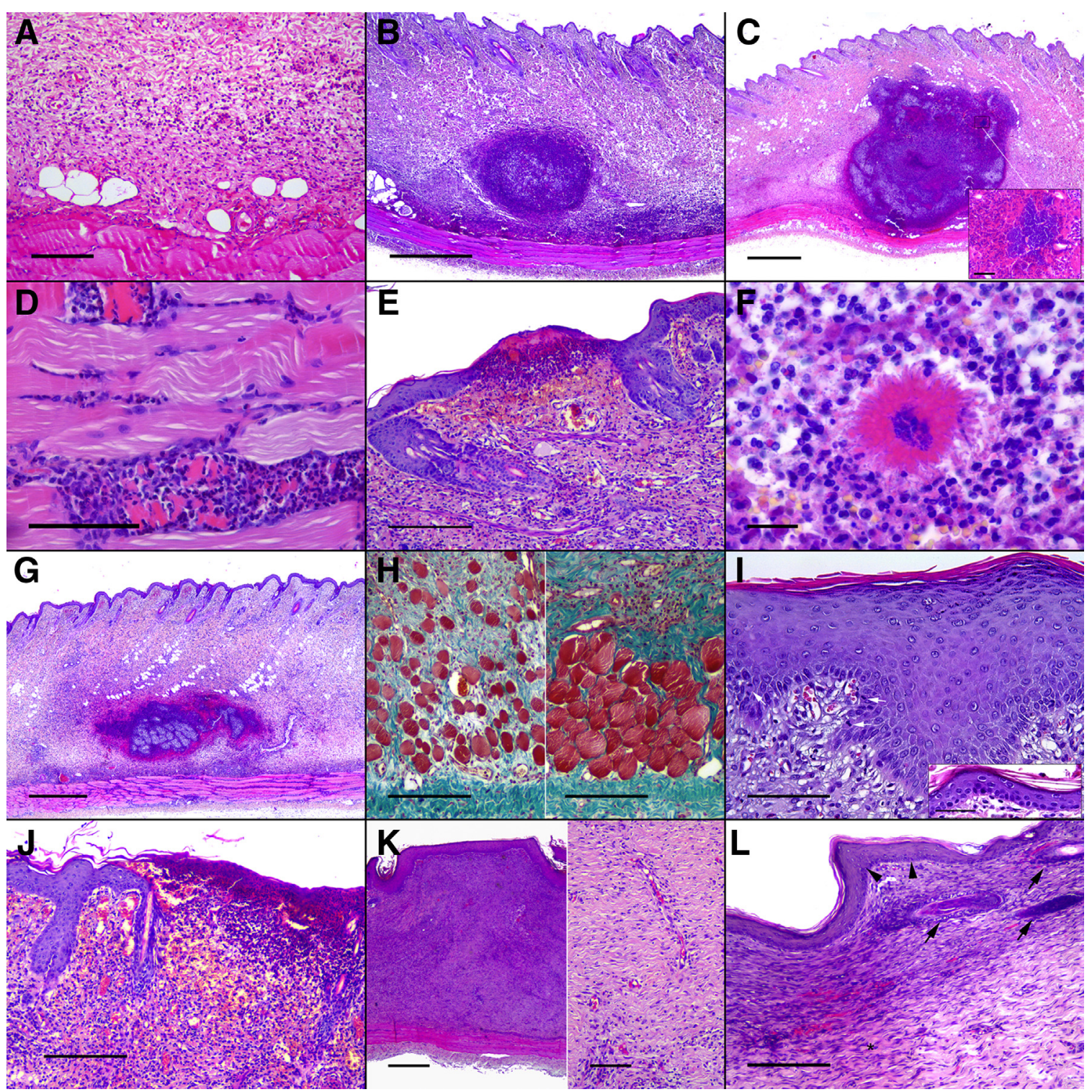

Figure 4 Different histologic characteristics of lesions throughout infection with the Jwt Staphylococcus aureus strain. A: At 0.5 days postinfection (dpi): Slight increase in the number of heterophils. Deep dermis is shown. Hematoxylin and eosin (H\&E) stain was used. B: At 1 dpi): Abscess surrounded by numerous diffusely distributed heterophils. Skin is shown. H\&E stain was used. C: At 2 dpi: Well-defined abscess characterized by a center of eosinophilic necrosis, with numerous coccoid bacteria inside (inset). Skin and bacteria (inset) are shown. H\&E stain was used. D: At 2 dpi: Muscular degeneration caused by the intense interstitial infiltration of heterophils. Cutaneous muscle is shown. H\&E stain was used. E: At 3 dpi: Necrosis of epidermis. Epidermis is shown. H\&E stain was used. F: At 3 dpi: A bacterial colony surrounded by star-shaped homogeneous eosinophilic material and an external layer of inflammatory cells. Splendore-Hoeppli phenomenon is shown. H\&E stain was used. G: At 3 dpi: Abscess internally composed of the fusion of several Splendore-Hoeppli phenomena, surrounded by a thin eosinophilic layer of necrosis. Skin is shown. H\&E stain was used. H: At 3 dpi: Severe atrophy of myocytes, infiltrated by connective tissue (left panel) versus normal muscular tissue (right panel). Cutaneous muscle is shown. Masson trichrome stain was used. I: At 7 dpi: Significant increase in the number of cells with numerous mitosis (arrows) on the basal layer of the epidermis (epidermal hyperplasia or acanthosis), compared with normal epidermis (inset). Epidermis is shown. H\&E stain was used. J: At 14 dpi: Severe inflammatory reaction associated with an ulcer and epidermal hyperplasia on the border. Epidermis is shown. H\&E stain was used. K: At 14 dpi: Repair of the lesion characterized by acanthosis, absence of skin adnexa (left panel), and presence of abundant granulation tissue composed of numerous vessels and fibrous tissue (right panel). Skin (left panel) and deep dermis (right panel) are shown. H\&E stain was used. L: At 28 dpi: Epithelial hyperplasia (arrowheads) and dermis with abundant fibrous tissue, blood vessels, scarce heterophils, and some small hemorrhages (asterisk). The periphery of the lesion is invaded by hair follicles (arrows). Epidermis and dermis are shown. H\&E stain was used. Scale bars: $200 \mu \mathrm{m}(\mathbf{A}, \mathbf{E}, \mathbf{H}, \mathbf{J}, \mathbf{K}$, right panel, and $\mathbf{L}) ; 1 \mathrm{~mm}(\mathbf{B}, \mathbf{C}$, main image, and G); $50 \mu \mathrm{m}(\mathbf{C}$ and I, insets); $100 \mu \mathrm{m}(\mathbf{D}$ and $\mathbf{I}$, main image); $20 \mu \mathrm{m}(\mathbf{F}) ; 500 \mu \mathrm{m}$ (K, left panel).

in some cases, was still interrupted by inflammatory cells and atrophied (Figure $1 \mathrm{H}$ ).

Finally, on $28 \mathrm{dpi}$, the epidermis of all the animals showed hyperplasia, but with a thinner epithelium than on
21 dpi (1.5-fold thicker than normal). Skin adnexa were absent in the central lesion, but the periphery was invaded by hair follicles as a consequence of scar retraction (Figure 1I). The dermis presented mature granulation tissue 


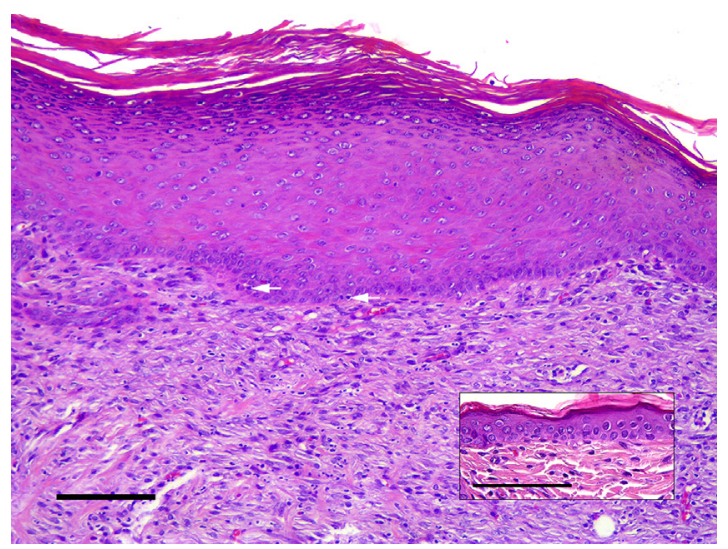

Figure 5 Hyperplasia of the epithelium with the presence of numerous mitoses in the basal layer (arrows) on 21 days postinfection with the Jwt Staphylococcus aureus strain. Inset: Normal epithelium. Hematoxylin and eosin stain. Scale bars $=100 \mu \mathrm{m}$ (main image and inset).

with abundant fibrous tissue and blood vessels, practically without erythrocytes and with scarce heterophils, as well as some small hemorrhages (Figure 4L). Cutaneous muscle had completely regenerated.

\section{Immunohistochemical Studies}

Variations in the counts of the studied cells (T-lymphocyte $\mathrm{CD}^{+}$, plasma cell $\mathrm{IgG}^{+}$, and macrophage $\mathrm{RAM}_{1} 1^{+}$) were detected locally in the inoculation area during the experiment. The T-lymphocyte $\left(\mathrm{CD}^{+}\right)$counts increased during the experiment until $21 \mathrm{dpi}\left(17.55 \pm 1.90\right.$ cells $\left./ \mathrm{mm}^{2}\right)$ and then abruptly decreased on 28 dpi (Figure 6A). Plasma cells $\left(\mathrm{IgG}^{+}\right)$appeared in small groups, mainly in the deep dermis. They were few in number in lesions in the acute inflammation phase (first 3 days), but significantly increased on 7 dpi and remained high on 14 and 21 dpi $(11.03,11.78$, and $11.15 \pm 1.93$ cells $/ \mathrm{mm}^{2}$, respectively; $P<0.001$ ), and once again abruptly decreased on 28 dpi (Figure 6B). The macrophages counts $\left(\mathrm{RAM} 11^{+}\right)$remained with no differences compared with 0 dpi until 3 dpi when they significantly increased until $14 \mathrm{dpi}$. At this time, the number of macrophages reached the highest level $\left(10.75 \pm 1.16\right.$ cells $/ \mathrm{mm}^{2}$; $P<0.001$ ) before lowering on $21 \mathrm{dpi}$ (still higher than on $0 \mathrm{dpi}$ ) and $28 \mathrm{dpi}$ (Figure 6C). Macrophages were diffusely located in the inoculation area when no abscess was present, but when abscesses started organizing, macrophages were located around them and formed a palisade on the outer abscess layer. No significant differences were detected in the number of B-lymphocyte CD79 $\alpha^{+}$.

\section{Flow Cytometer Results}

Figure 5 shows the results of the flow cytometric analysis of the peripheral blood cells in the rabbits infected by $S$. aureus Jwt. Intradermal inoculation induced modifications in the counts of most leukocyte populations (granulocytes, monocytes, and total $\mathrm{B}$ and $\mathrm{T} \mathrm{CD} 25^{+}$lymphocytes). Total leukocyte (Figure 6D) and granulocyte (Figure 6F) counts showed two peaks during the study: a first sharp increase on
$1 \mathrm{dpi}$ and a second greater increase from 7 to $14 \mathrm{dpi}$, which decreased on $21 \mathrm{dpi}$ (more abruptly for granulocytes). The number of monocytes (Figure $6 \mathrm{G}$ ) gradually increased from 3 to $14 \mathrm{dpi}$, when its highest value was reached $(869.5 \times$ $\left.10^{6} / \mathrm{L} ; P<0.001\right)$. The number of total lymphocytes increased from 0 to $2 \mathrm{dpi}$, when counts were kept elevated until the end of the experiment (Figure 6E). Regarding the lymphocyte subpopulations, statistical differences were observed only in the B (Figure 6H) and CD25 (Figure 6I) lymphocytes. B cells had higher values at 0.5 , and especially at 21 , dpi compared with 0 dpi (43.29 and $81.66 \times$ $10^{6} / \mathrm{L}$, respectively; $\left.P<0.001\right)$. The cell counts of the $\mathrm{CD} 25^{+}$lymphocytes abruptly increased on $0.5 \mathrm{dpi}$ and were 11.9-fold higher than on $0 \mathrm{dpi}$ and on later experimental days. No significant differences were observed in the number of $\mathrm{T}$ lymphocytes, $\mathrm{T} \mathrm{CD} 4^{+}$lymphocytes, and $\mathrm{T}$ $\mathrm{CD}^{+}$lymphocytes.

\section{Plasmatic and Tissue Cytokines}

Statistical differences were detected only in IL-4 and IL-18 in plasma and in IL-4 and IFN- $\gamma$ in skin samples (Table 6). The plasmatic levels of IL-4 and IL-18 oscillated throughout the experiment. Relative to $0 \mathrm{dpi}$, statistical differences were only detected for IL-4 on 28 dpi and IL-18 on 14 and 28 dpi, where these cytokines reached their highest plasmatic levels ( $8.9 \mathrm{pg} \mathrm{IL}-4 / \mathrm{mL}$ and $463.4 \mathrm{pg} \mathrm{IL}-18 / \mathrm{mL}$ ) on $28 \mathrm{dpi}$. Similar to plasma, the IL-4 detected in skin tissue significantly increased on $28 \mathrm{dpi}(15.5 \mathrm{pg} / \mathrm{mL} ; P<0.05)$. Conversely, the IFN- $\gamma$ level in tissues abruptly increased on 0.5 dpi $(14,015 \pm 1804 \mathrm{pg} / \mathrm{mL} ; P<0.05)$ before returning to previous levels. No significant differences were observed in IL-1 $\beta$ and IL-17 levels.

\section{Study 1b: Effects of the Selected Genes and the Global Regulator Involved in Abscess Development}

Once the lesions and peripheral and local immune responses had been described, the aim of this section was to evaluate the contribution of different isogenic mutants of the Jwt strain (J $\Delta a g r, \mathrm{~J} \Delta \operatorname{coa} \Delta v w b, \mathrm{~J} \Delta h l a$, and $\mathrm{J} \Delta p s m \alpha$ ) to the development and characteristics of abscesses on 7 dpi using the proposed rabbit skin model. Unexpectedly, all of the mutant strains were able to generate lesions like the wild type in terms of degree of severity (Figure 7). Whenever present, lesions were macroscopically indistinguishable from those generated by the wild-type strain.

At the histologic level, Splendore-Hoeppli phenomena were observed with all the strains, but not with the same incidence. Only statistically differences were observed with $\mathrm{J} \Delta$ hla $(85 \% \mathrm{Jwt}$ versus $68 \% \mathrm{~J} \Delta$ hla $; P<0.05$ ) (Figure $8 \mathrm{~A}$ ). The percentage of distributed heterophiles surrounding the abscesses was higher in the mutant strains with respect to Jwt (20\% for $\mathrm{J} \Delta c o a \Delta v w b, 50 \%$ for $\mathrm{J} \Delta h l a$, and $30 \%$ for $\mathrm{J} \Delta p s m \alpha$ and $\mathrm{J} \Delta a g r ; P<0.05$ ) (Figure $8 \mathrm{~B}$ ). 

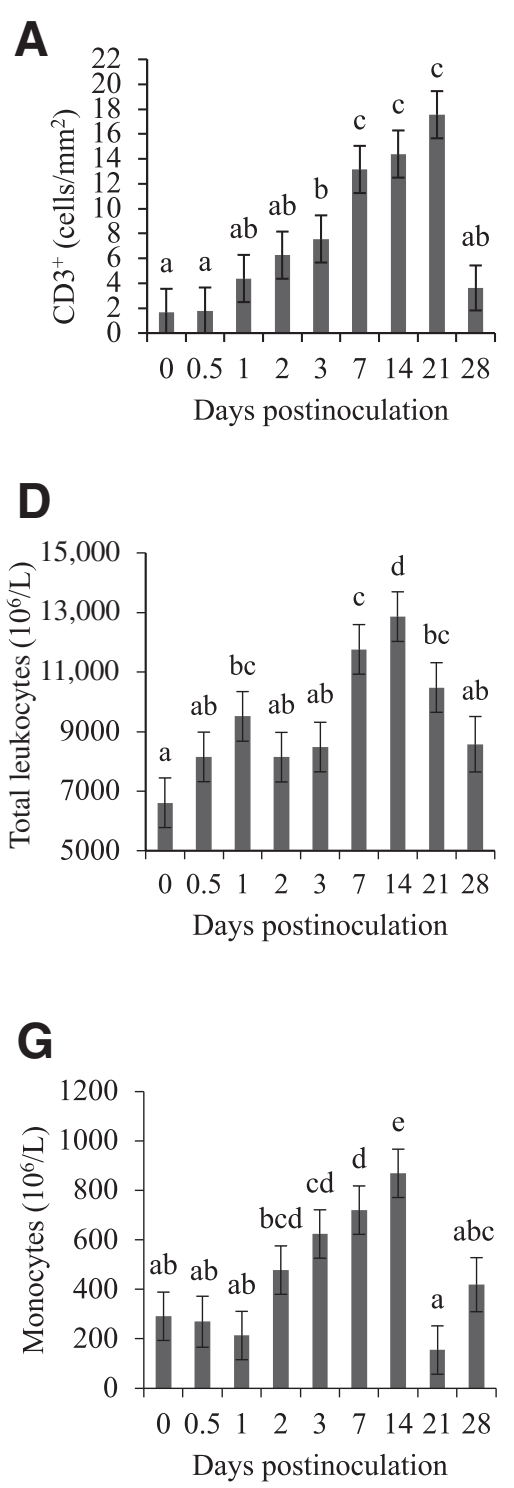

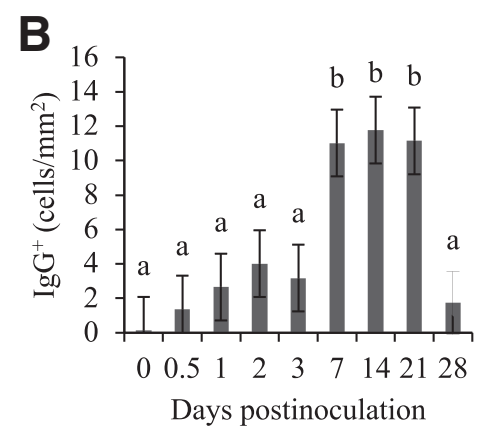

E
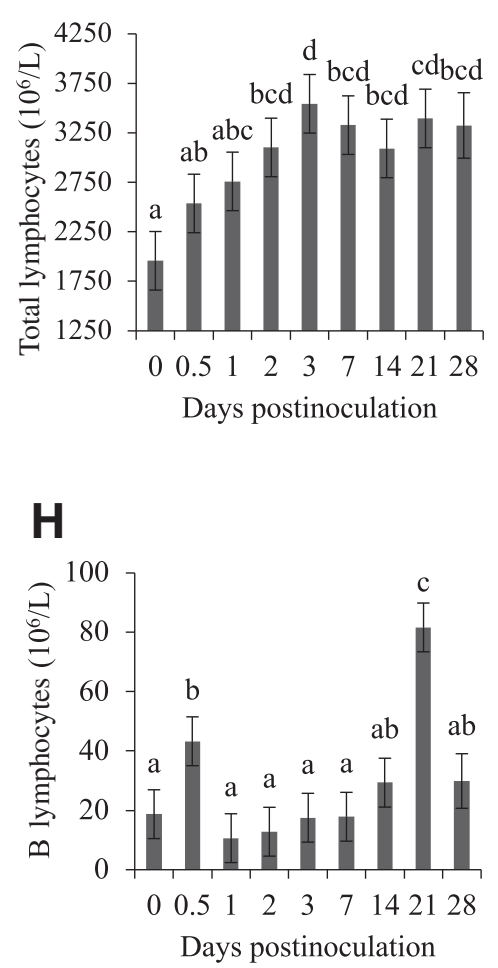
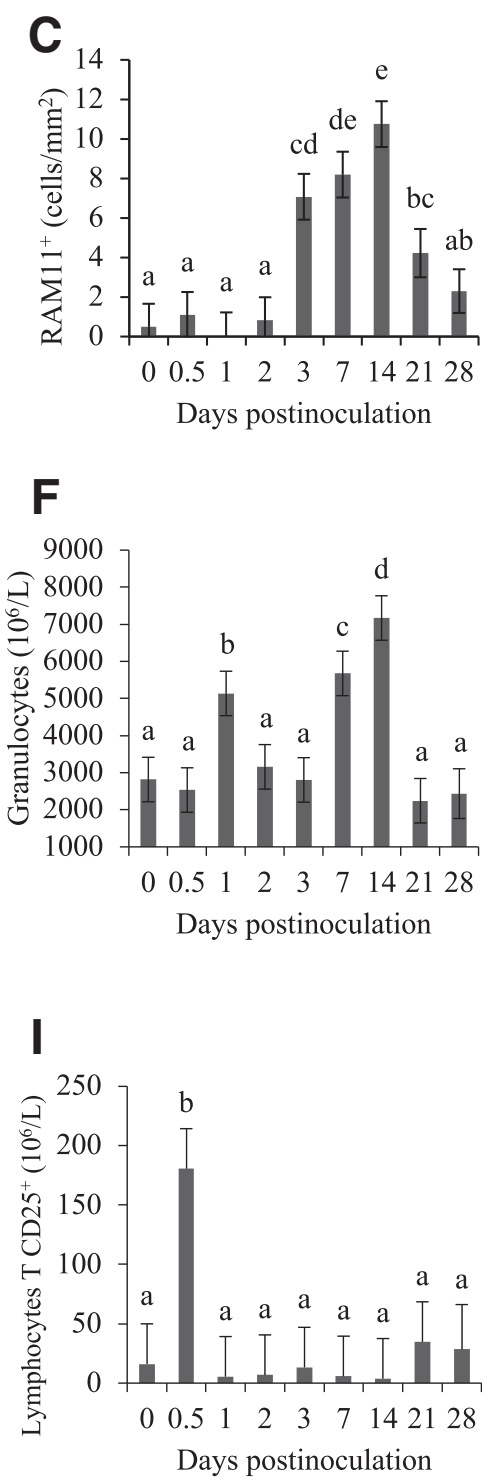

Figure 6 Evolution of the counts of $\mathrm{CD}^{+}(\mathbf{A}), \mathrm{IgG}^{+}(\mathbf{B})$, and $\mathrm{RAM}_{1}{ }^{+}(\mathbf{C})$ cells immunochemically marked in the skin samples and total leukocytes (D), total lymphocytes $(\mathbf{E})$, granulocytes $(\mathbf{F})$, monocytes $(\mathbf{G})$, lymphocyte $B(\mathbf{H})$, and lymphocyte $\operatorname{TCD}^{+} 5^{+}(\mathbf{I})\left(\times 10^{6} / \mathrm{L}\right)$ in peripheral blood after intradermal inoculation with the Jwt Staphylococcus aureus strain. Error bars correspond to the SEM for each least square mean $(\mathbf{A}-\mathbf{I}) . n=10$ animals per day $(\mathbf{A}-\mathbf{I}) .{ }^{a-e}$ The means that do not share a superscript in the same figure significantly differ for each postinoculation time $(P<0.05)$.

All the lesions caused by each studied strain (the wild type and mutants) presented an eosinophilic layer surrounding abscesses on 7 dpi. However, there were differences in the characteristics of this band. The percentage of complete eosinophilic layers always was lower for mutants than for $\mathrm{Jwt}$ ( $-30 \%$ for $\mathrm{J} \Delta \operatorname{coa} \Delta v w b,-90 \%$ for $\mathrm{J} \Delta h l a,-45 \%$ for $\mathrm{J} \Delta$ psm $\alpha$, and $-50 \%$ for $\mathrm{J} \Delta a g r ; P<0.05$ ) (Figure $8 \mathrm{C}$ ). These histologic differences are related to the degree of maturation of the abscess. Thus, the lesions generated by the mutants at 7 dpi resembled those presented by the wild-type strain in 2 to $3 \mathrm{dpi}$, which could be interpreted as the absence of these genes delays the development of the lesions.
Study 2: Comparison of the Lesions Caused by a Human Rabbitized Strain and Different Rabbit Strains of Known Virulence

Once the infection model had been characterized and that all the rabbit strains were capable of generating lesions at low doses had been verified, our intention was to test the model with a human strain. The inconvenience herein is that human S. aureus strains (eg, F strain) do not cause lesions at low doses (300 CFUs) in rabbits. ${ }^{13}$ In fact, to be able to produce them, it is necessary to use high bacterial doses ( $\left.>10^{4} \mathrm{CFUs}\right)$ and, when this occurs, the evolution of lesions (faster) and the produced characteristics (eg, presence of extensive 
Table 6 Evolution of the Concentration $(\mathrm{pg} / \mathrm{mL})$ of Cytokines on Plasma and Skin Tissue after the Intradermal Inoculation with the Jwt Staphylococcus aureus Strain ( $n=10$ Animals per Day)

\begin{tabular}{|c|c|c|c|c|c|c|c|c|c|c|c|}
\hline \multirow[b]{2}{*}{ Variable } & \multicolumn{9}{|c|}{ Postinoculation time, days } & \multirow[b]{2}{*}{ SEM } & \multirow[b]{2}{*}{$P$ value } \\
\hline & 0 & 0.5 & 1 & 2 & 3 & 7 & 14 & 21 & 28 & & \\
\hline \multicolumn{12}{|l|}{ Plasma } \\
\hline $\mathrm{IL}-18$ & $126.8^{\mathrm{ab}}$ & $316.4^{b c}$ & $263.0^{b c}$ & $245.9^{b}$ & $328.2^{b c}$ & $87.2^{\mathrm{a}}$ & $350.0^{c}$ & $307.2^{b c}$ & $463.4^{\mathrm{d}}$ & 39.8 & $<0.001$ \\
\hline \multicolumn{12}{|l|}{ Skin } \\
\hline IL-4 & $8.998^{a}$ & $6.419^{\mathrm{a}}$ & $7.080^{\mathrm{a}}$ & $7.780^{\mathrm{a}}$ & $9.392^{\mathrm{a}}$ & $7.307^{\mathrm{a}}$ & $8.774^{\mathrm{a}}$ & $9.951^{a}$ & $15.479^{b}$ & 1.554 & 0.001 \\
\hline
\end{tabular}

For a given trait presented in a row, $P$ value tests hypothesis H0: there was no effect of treatment to explain the observed trait variance.

${ }^{\mathrm{a}-\mathrm{d}}$ The means in a same row not sharing superscript significantly differ at $P<0.05$ for day postinoculation.

IFN $-\gamma$, interferon- $\gamma$.

coagulative necrosis, thrombosis, absence of SplendoreHoeppli phenomena, or peripheral eosinophilic layers on 3 dpi) differ vastly from those observed by rabbit strains at low doses (J.M.C., unpublished data) (Supplemental Figure S1). Therefore, we had to use a human strain adapted to rabbits $\left(\mathrm{Fdlt} \mathrm{B}^{\mathrm{r}}\right)$, which we had previously demonstrated was able to infect this species at low doses. ${ }^{13}$

The specific aim was to compare the response to infection after inoculating the $\mathrm{Fdlt}_{\mathrm{B}}{ }^{\mathrm{r}}$ strain with $S$. aureus strains obtained from rabbits of known virulence, such as Jwt (high virulence), $\mathrm{Jrot}^{+}$(medium virulence), and $\mathrm{Jdlt}_{\mathrm{B}}^{\mathrm{h}}$ (low virulence), using the model described in Study 1 to demonstrate its utility with strains of different origins.

\section{Health Parameters}

No animal experienced fever, and no differences in temperature between the studied groups were observed.

\section{Gross Lesions}

Differences in both the number of infected animals and the characteristics of their lesions after inoculation with the studied S. aureus strains were detected. Although 100\% of the rabbits were infected by human $F d l t \mathrm{~B}^{\mathrm{r}}$ and rabbit Jwt,
$76.7 \%$ and $60 \%$ of the animals inoculated with $\mathrm{Jrot}^{+}$and $\mathrm{J} d l t \mathrm{~B}^{\mathrm{h}}$ showed lesions, respectively. The severity of lesions caused by $\mathrm{Fdlt}_{\mathrm{B}} \mathrm{r}^{\mathrm{r}}$ was intermediate, between Jwt (more severe lesions) and $\mathrm{Jrot}^{+}$, and $\mathrm{Jdlt}_{\mathrm{B}}^{\mathrm{h}}$ (milder lesions) (Figure 9).

On $1 \mathrm{dpi}$, the inoculation points showed erythema in $100 \%$ of the animals infected by $\mathrm{Fdlt}_{\mathrm{B}}{ }^{\mathrm{r}}$, Jwt, and $\mathrm{Jrot}^{+}$and $80 \%$ in the rabbits inoculated with $\mathrm{Fdlt}_{\mathrm{B}}{ }^{\mathrm{r}}$. On 3 dpi, lesions evolved to nodules in $100 \%$ of the animals infected by the $\mathrm{Fdlt}_{\mathrm{B}}^{\mathrm{r}}$ and Jwt strains, but only in $30 \%$ of the rabbits inoculated with $\mathrm{Jrot}^{+}$and $10 \%$ with $\mathrm{J} d l t \mathrm{~B}{ }^{\mathrm{h}}$. At the end of the experiment ( $7 \mathrm{dpi}$ ), although all the animals infected with $\mathrm{Fdlt} \mathrm{B}^{\mathrm{r}}$ and Jwt presented nodules, and even some developed dermonecrosis (10\% and 50\%, respectively), only $50 \%$ of the rabbits inoculated with $\mathrm{Jrot}^{+}$and $\mathrm{J} d l t \mathrm{~B}^{\mathrm{h}}$ showed lesions. The animals infected with $\mathrm{Jrot}^{+}$ exhibited erythema and nodules $(40 \%)$, but erythema was observed only in the rabbits infected with $\mathrm{J} d l t \mathrm{~B}^{\mathrm{h}}$ at this time point.

\section{Bacteriology}

The bacterial counts obtained from the animals inoculated with $\mathrm{FdltB}^{\mathrm{r}}$ and $\mathrm{Jrot}^{+}$followed the same dynamics as the

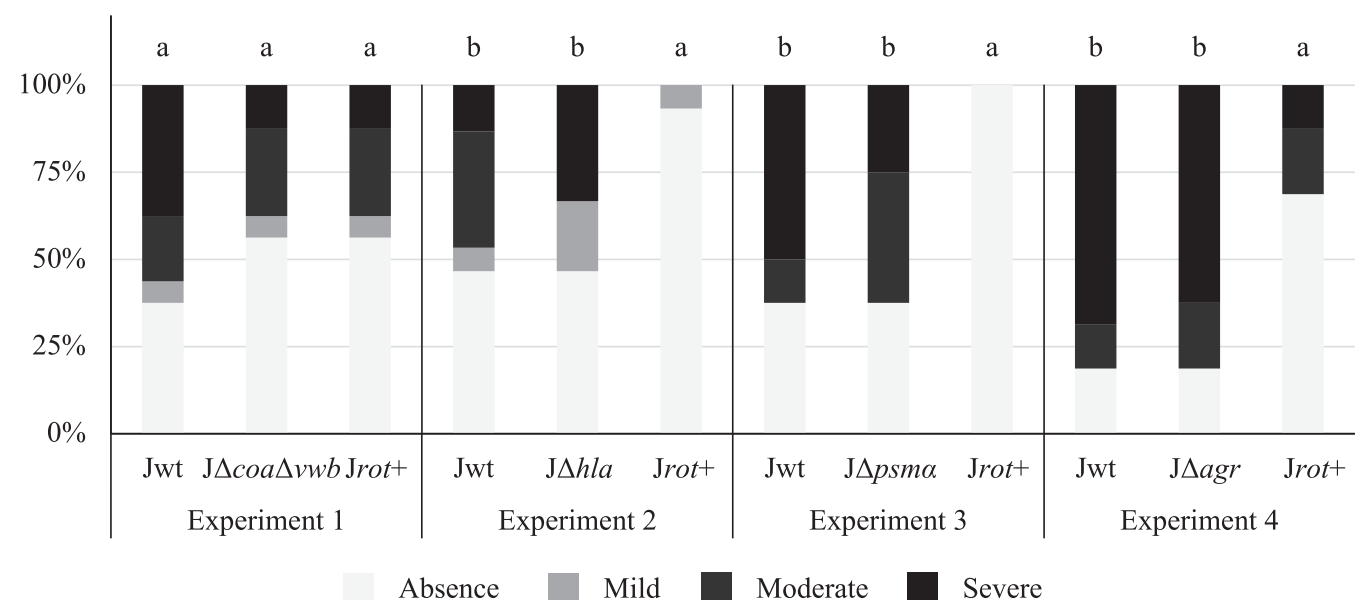

Figure 7 Severity assessment of gross lesions on day 7 after intradermal inoculation with different mutants (J $\Delta$ coa $\Delta v w b, \mathrm{~J} \Delta h l a, \mathrm{~J} \Delta p s m \alpha$, and $\mathrm{J} \Delta a g r)$ versus Jwt (high virulent) and Jrot ${ }^{+}$(low virulent) strains of Staphylococcus aureus. $n=16 \mathrm{~J} \Delta$ coa $\Delta v w b ; n=15 \mathrm{~J} \Delta h l a$ and J $\Delta a g r ; n=8 \mathrm{~J} \Delta p s m \alpha$. ${ }^{\text {a,b }}$ Within an experiment, the groups not sharing letters above the bar significantly differ $(P<0.05)$. 


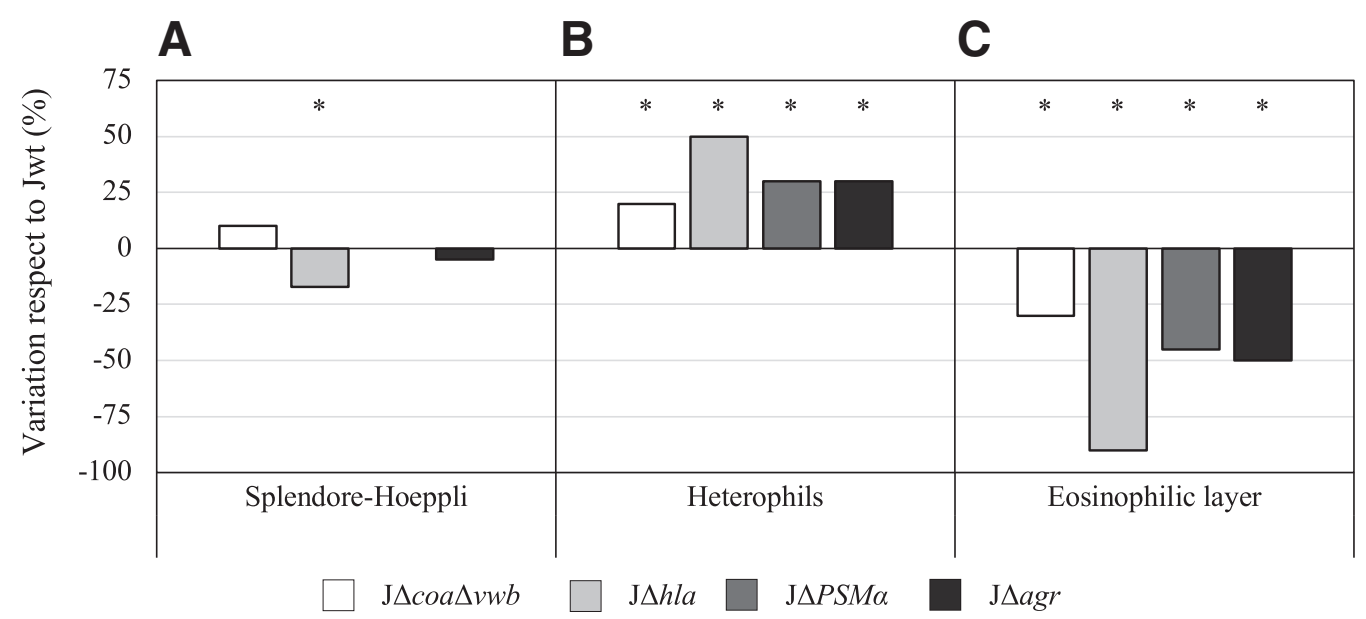

Figure 8 Presence of the main histologic characteristics (Splendore-Hoeppli phenomena; A), heterophils surrounding the abscesses (B), and complete eosinophilic layers (C) on day 7 after intradermal inoculation with different mutant strains (J $\Delta$ coa $\Delta v w b, \mathrm{~J} \Delta h l a, \mathrm{~J} \Delta p s m \alpha$, and J $\Delta$ agr) of Staphylococcus aureus. Evaluated as variation in relation to intradermal inoculation with Jwt for: $\mathrm{J} \Delta$ coa $\Delta v w b, \mathrm{~J} \Delta h l a, \mathrm{~J} \Delta p s m \alpha$, and J $\Delta a g r . n=16 \mathrm{~J} \Delta$ coa $\Delta v w b ; n=15 \mathrm{~J} \Delta h l a$ and $\mathrm{J} \Delta$ agr $; n=8 \mathrm{~J} \Delta$ psm $\alpha .{ }^{*} P<0.05$ versus intradermal inoculation with Jwt.

Jwt strain on 1, 3, and 7 dpi (Figure 10), but the average number of bacteria obtained from their lesions was smaller $\left(6.18 \pm 32.21 \times 10^{6}\right.$ and $1.10 \pm 47.54 \times 10^{6} \mathrm{CFUs} / \mathrm{g}$, respectively; $P<0.05)$ than from the lesions caused by Jwt $\left(244.36 \pm 36.98 \times 10^{6} \mathrm{CFUs} / \mathrm{g}\right)$. Bacteria were isolated only in one animal infected by $\mathrm{J} d l t \mathrm{~B}^{\mathrm{h}}$ on $3 \mathrm{dpi}\left(2.26 \times 10^{3}\right.$ CFUs/g) at 3 dpi. All the cultured and identified bacteria recovered from lesions were the same as those inoculated, and no bacteria were isolated from blood or kidneys.

\section{Histologic Findings}

Different degrees of severity were observed in the histologic lesions, depending on the type of inoculated strain (Table 7). In general, $\mathrm{F} d l t \mathrm{~B}^{\mathrm{r}}$ caused more similar (but milder) lesions to the Jwt strain than to the other two. Vascular dilatation and edema in the superficial dermis observed in the animals infected by $\mathrm{F} d l t \mathrm{~B}^{\mathrm{r}}$ were more severe than $\mathrm{Jrot}^{+}$and resembled Jwt.

The abscesses caused by $\mathrm{F} d l t \mathrm{~B}^{\mathrm{r}}$ were evident on $1 \mathrm{dpi}$ and were more numerous and more severe on $3 \mathrm{dpi}$, similarly to Jwt. Conversely, the severity of the abscesses (heterophils organized) (Table 7) in the animals infected by $\mathrm{Jrot}^{+}$and $\mathrm{J} d l t \mathrm{~B}^{\mathrm{h}}$ reduced on 3 dpi. In this last case, abscesses were classified mainly as mild (Figure 11A).

The abscesses produced by the three mutant strains were histologically more immature compared with Jwt as they did not show a complete eosinophilic peri-abscess layer. On 3 dpi, all the Jwt-related abscesses had a well-developed eosinophilic layer, only $55 \%$ of $\mathrm{F} d l t \mathrm{~B}^{\mathrm{r}}$ and $20 \%$ of $\mathrm{Jrot}^{+}$had one, and no band was detected in the $\mathrm{J} d l t \mathrm{~B}^{\mathrm{h}}$ abscesses. At the end of the

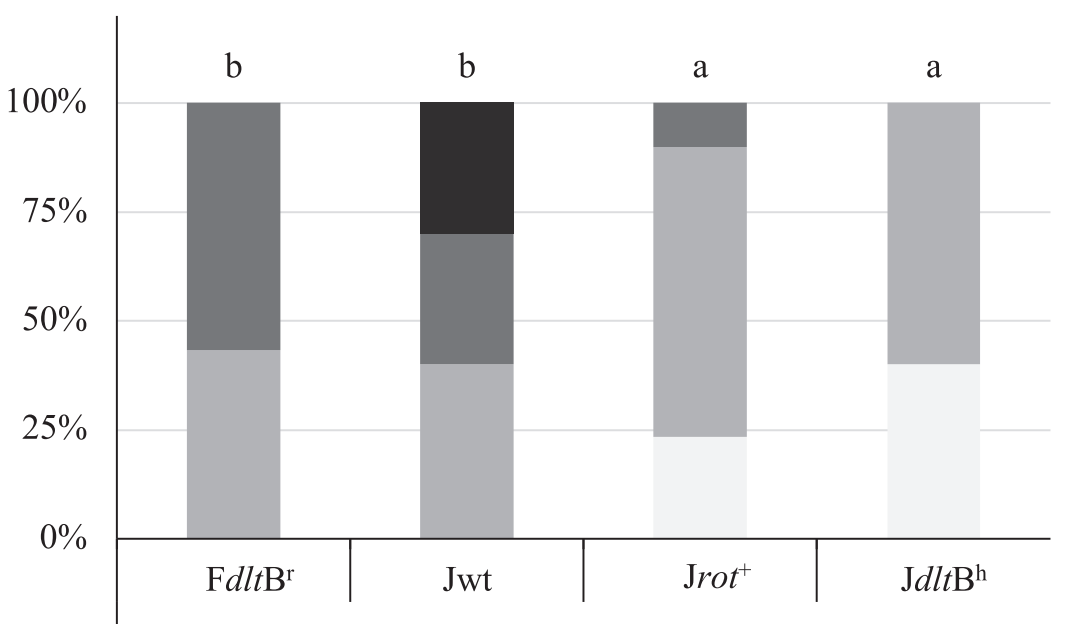

Figure 9 Mean severity assessment of gross lesions after intradermal inoculation with different strains (Fdlt $\mathrm{B}^{\mathrm{r}}$, Jwt, Jrot ${ }^{+}$, and Jdlt $\mathrm{B}^{\mathrm{h}}$ ) of Staphylococcus aureus. Assessment is represented as the percentage at four levels: absence, mild, moderate, or severe. $n=30$ animals per strain. ${ }^{a, b}$ The groups not sharing letters above the bar significantly differ $(P<0.05)$. 


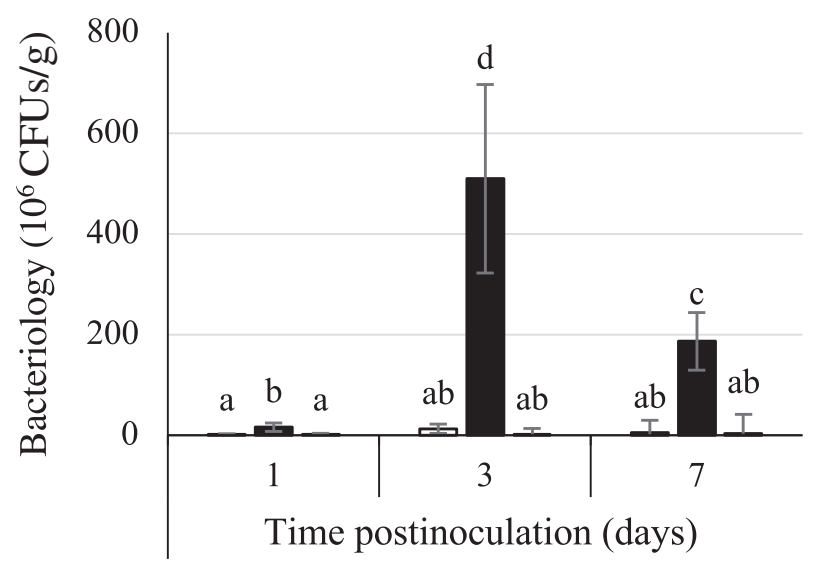

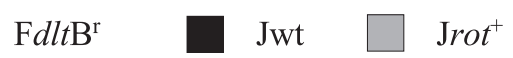

Figure 10 Colony-forming units (CFUs) after intradermal inoculation with different strains of Staphylococcus aureus ( $\mathrm{FdltB}^{\mathrm{r}}$, Jwt, and Jrot ${ }^{+}$). Error bars correspond to the SEM for each least square mean. $n=10$ animals per strain and day. ${ }^{\mathrm{a}-\mathrm{d}}$ The means not sharing superscript significantly differ $(P<0.05)$.

experiment (7 dpi), percentages varied, but differences remained (100\% Jwt, 36\% $\mathrm{F} d l t \mathrm{~B}^{\mathrm{r}}, 30 \% \mathrm{Jrot}^{+}$, and $\left.0 \% \mathrm{~J} d l t \mathrm{~B}^{\mathrm{h}}\right)$.

Splendore-Hoeppli phenomena were seen only in the abscesses from the rabbits infected by Jwt and $\mathrm{Jrot}^{+}$on 3 and 7 dpi. There were fewer Splendore-Hoeppli phenomena in $\mathrm{Jrot}^{+}$
( 1 on $3 \mathrm{dpi}$ and 2 on $7 \mathrm{dpi}$ ) than in Jwt ( 5 on $3 \mathrm{dpi}$ and 8 on $7 \mathrm{dpi}$ ), although the number of abscesses also lowered with Jrot $+(2$ on $3 \mathrm{dpi}$ and 3 on $7 \mathrm{dpi}$ ) than with Jwt (10 at both time points).

The degree of dilated blood vessels in the deeper dermis close to cutaneous muscle was higher in the animals inoculated with strains $\mathrm{F} d l t \mathrm{~B}^{\mathrm{r}}$ and $\mathrm{J} d l t \mathrm{~B}^{\mathrm{h}}$ for all of the studied times, but the incidence of perivascular inflammation was higher with Jwt $(P<0.05)$ (Table 7).

The pathologic findings (inflammation and degeneration) observed in cutaneous muscle were similar between strains $\mathrm{F} d l t \mathrm{~B}^{\mathrm{r}}$ and Jwt, and were more severe than those caused by strains $\mathrm{Jrot}^{+}$and $\mathrm{J} d l t \mathrm{~B}^{\mathrm{h}}(P<0.05)($ Table 7$)$.

\section{Immunohistochemical Studies}

The number of positive cells was related to lesion severity, with no positive cells in the animals without lesions regardless of the inoculated strain. The number of studied cells in the lesions caused by $\mathrm{F} d l t \mathrm{~B}^{\mathrm{r}}$ strain infection was lower to those of the wild-type strain (Jwt) and more similar to those of strain $\mathrm{Jrot}^{+}$and $\mathrm{J} d l t \mathrm{~B}^{\mathrm{h}}$ (Figure 11, B-E). The Tlymphocyte $\left(\mathrm{CD}^{+}\right)$counts were significantly higher during the three moments analyzed in the Jwt compared with $\mathrm{F} d l t \mathrm{~B}^{\mathrm{r}}$. The macrophage counts $\left(\mathrm{RAM} 11^{+}\right)$were significantly higher on 3 and 7 dpi in the animals infected with Jwt regarding to $\mathrm{F} d l t \mathrm{~B}^{\mathrm{r}}$, and the counts of $\mathrm{B}$ lymphocytes $\left(\mathrm{CD} 79 \alpha^{+}\right)$and plasma cells $\left(\mathrm{IgG}^{+}\right)$were significantly

Table 7 The Histopathologic Findings Observed after the Inoculation of Different Staphylococcus aureus Strains

\begin{tabular}{|c|c|c|c|c|c|c|}
\hline \multirow[b]{2}{*}{ Variable } & \multicolumn{4}{|l|}{ S. aureus strain } & \multicolumn{2}{|l|}{$P$ value } \\
\hline & $\overline{F d l t B^{r}}$ & Jwt & Jrot $^{+}$ & $J d l t B^{h}$ & Strain & Time $\times$ strain \\
\hline \multicolumn{7}{|l|}{ Epidermis* } \\
\hline Re-epithelization (mitosis) & $100 / 0 / 0 / 0$ & $100 / 0 / 0 / 0$ & $100 / 0 / 0 / 0$ & $100 / 0 / 0 / 0$ & 1.000 & 1.000 \\
\hline \multicolumn{7}{|l|}{ Superficial dermis } \\
\hline Vascular dilatation & $44 / 52 / 4 / 0^{b}$ & $16 / 42 / 26 / 16^{c}$ & $40 / 57 / 3 / 0^{a}$ & $33 / 67 / 0 / 0^{\mathrm{ab}}$ & 0.001 & 0.001 \\
\hline \multicolumn{7}{|l|}{ Deep dermis } \\
\hline Vascular dilatation & $2 / 46 / 52 / 0^{b}$ & $0 / 84 / 13 / 3^{a}$ & $0 / 77 / 13 / 10^{a}$ & $0 / 41 / 56 / 3^{b}$ & 0.001 & 0.012 \\
\hline Perivascular inflammation & $2 / 46 / 52 / 0^{a}$ & $0 / 26 / 42 / 32^{b}$ & $77 / 7 / 16 / 0^{\mathrm{a}}$ & $3 / 47 / 50 / 0^{a}$ & 0.001 & 0.001 \\
\hline Heterophiles diffuse & $4 / 54 / 34 / 8^{a b}$ & $0 / 36 / 39 / 25^{b}$ & $0 / 64 / 23 / 13^{a}$ & $0 / 47 / 47 / 6^{a}$ & 0.016 & 0.060 \\
\hline Heterophiles organized (abscess) & $58 / 4 / 2 / 36^{b}$ & $6 / 4 / 3 / 87^{c}$ & $60 / 7 / 3 / 30^{\mathrm{a}}$ & $67 / 17 / 13 / 3^{a}$ & 0.001 & 0.001 \\
\hline Interstitial inflammation & $15 / 46 / 27 / 12^{b}$ & $3 / 14 / 42 / 41^{c}$ & $21 / 63 / 3 / 13^{a}$ & $17 / 53 / 27 / 3^{a}$ & 0.001 & 0.008 \\
\hline Atrophy/degeneration & $71 / 19 / 10 / 0^{b}$ & $35 / 35 / 30 / 0^{b}$ & $86 / 8 / 3 / 3^{a}$ & $100 / 0 / 0 / 0^{a}$ & 0.001 & 0.029 \\
\hline Hypertrophy & $100 / 0 / 0 / 0$ & $100 / 0 / 0 / 0$ & $97 / 3 / 0 / 0$ & $100 / 0 / 0 / 0$ & 0.415 & 1.000 \\
\hline Interruption & $96 / 2 / 2 / 0$ & $68 / 19 / 10 / 3$ & $97 / 0 / 0 / 3$ & $97 / 3 / 0 / 0$ & 0.191 & 0.525 \\
\hline Fibrosis & $100 / 0 / 0 / 0$ & $81 / 19 / 0 / 0$ & $100 / 0 / 0 / 0$ & $100 / 0 / 0 / 0$ & 0.066 & 0.144 \\
\hline
\end{tabular}

For a given trait presented in a row, $P$ value tests hypothesis H0: there was no effect of treatment to explain the observed trait variance. $n=10$ animals per day and strain.

${ }^{a-c}$ The means in a same row not sharing letters significantly differ $(P<0.05)$.

*Each histologic parameter presents four values $(\mathrm{w} / \mathrm{x} / \mathrm{y} / \mathrm{z})$ that indicate the average percentage of animals for the three sampling times (1, 3, and 7 days postinfection). The microscopic findings are classified as absent $(w)$, mild $(x)$, moderate $(y)$, and severe $(z)$. 
A

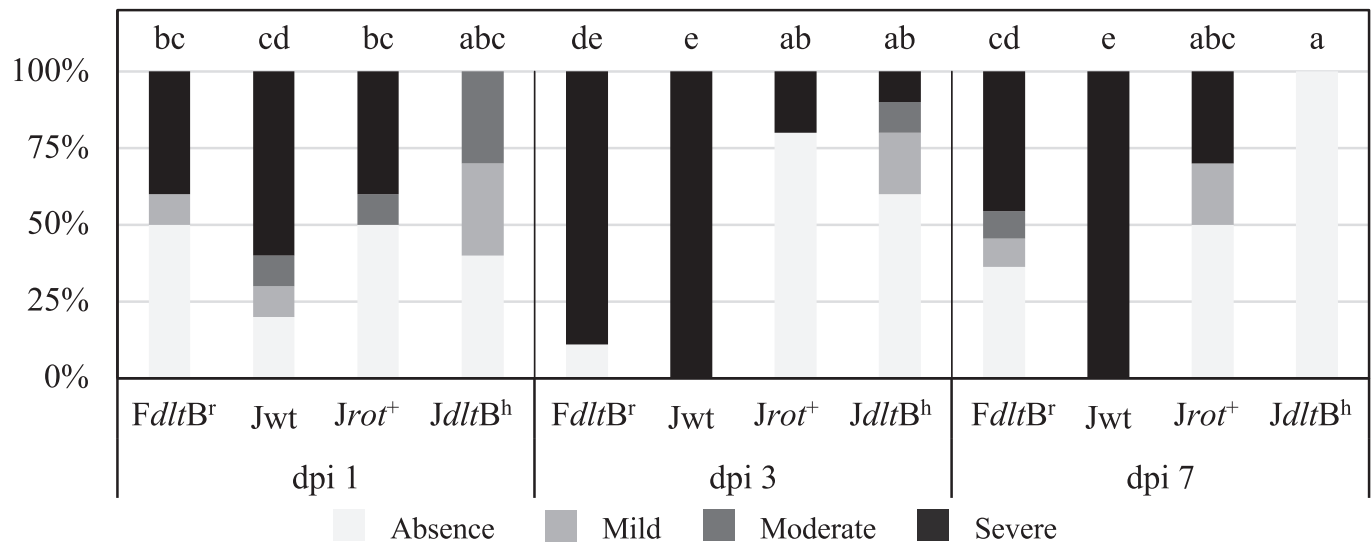

B

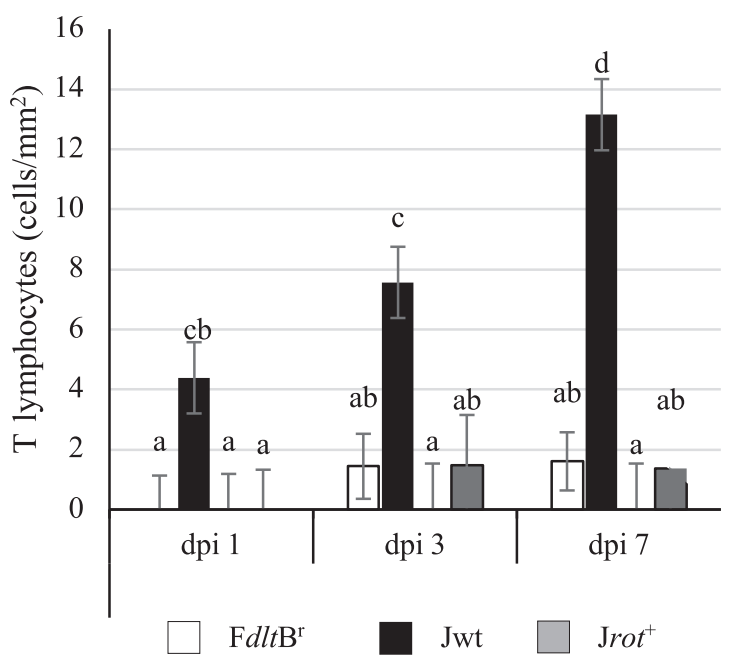

D

C
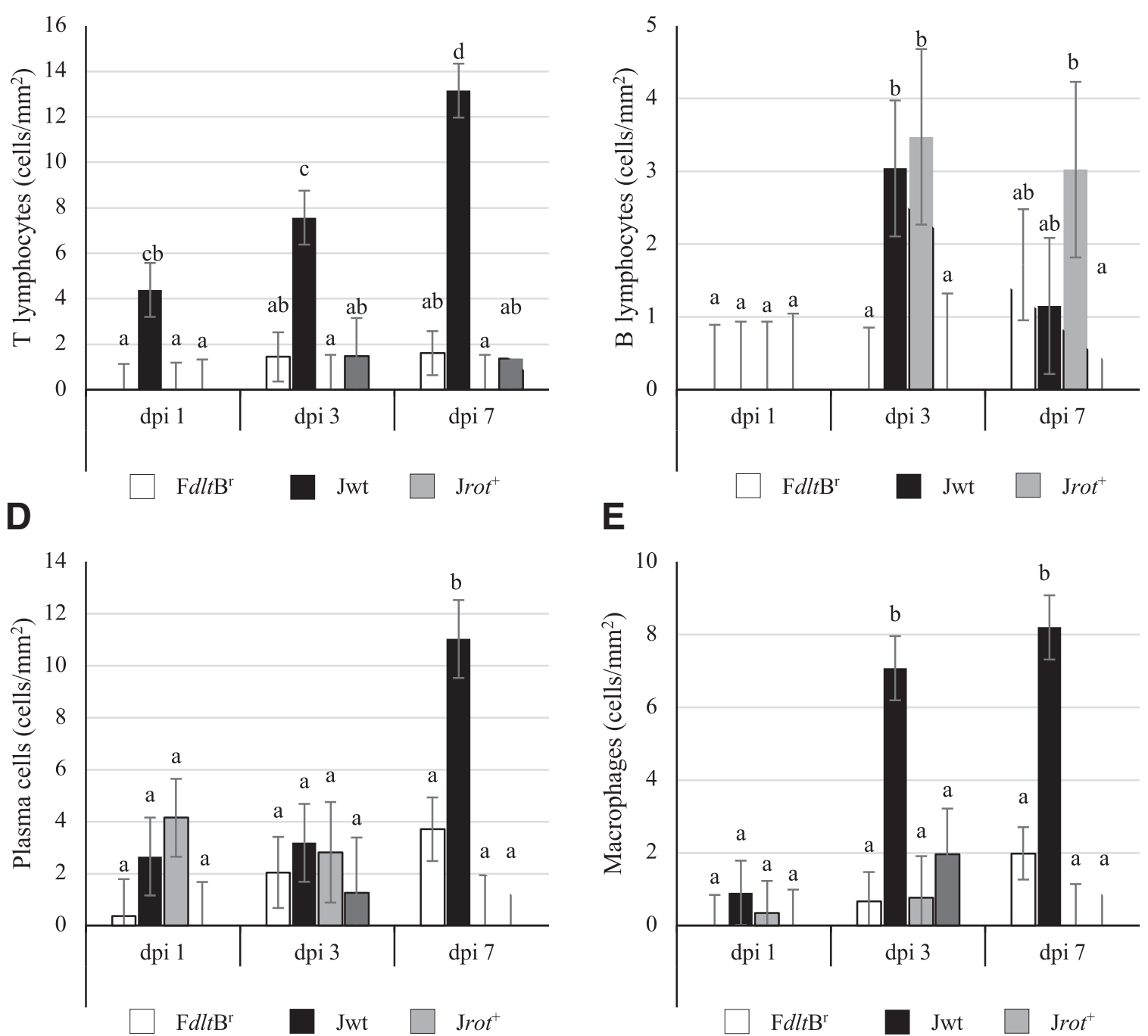

$\mathbf{E}$

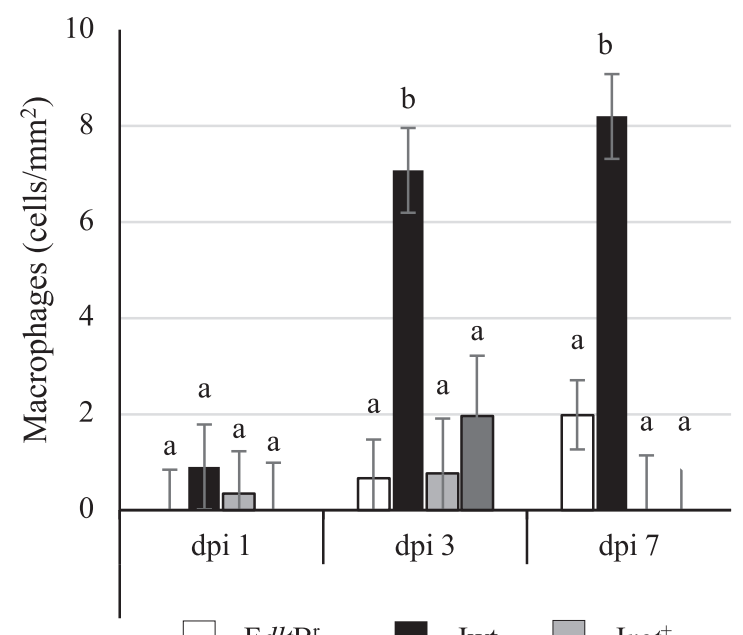

Figure 11 A: Severity assessment of abscesses (heterophils organized) histologically after infections with strains Fdlt $\mathrm{B}^{\mathrm{r}}$, Jwt, Jrot ${ }^{+}$, and Jdlt $\mathrm{B}^{\mathrm{h}}$ of Staphylococcus aureus. Assessment is represented at four levels: absence, mild, moderate, or severe. The groups not sharing letters ( $a, b, c, d, e)$ above the bar significantly differ. B-E: Evolution of the counts of T-lymphocyte $\mathrm{CD}^{+}(\mathbf{B}), \mathrm{B}$-lymphocyte $\mathrm{CD} 7 \alpha^{+}$(C), plasma cells positive for IgG ${ }^{+}(\mathbf{D})$, and macrophage

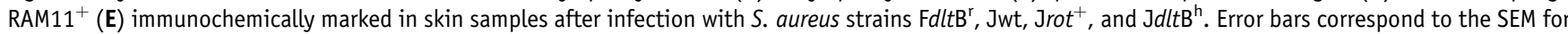
each least square mean (B-E). $n=30$ animals per strain $(\mathbf{A}) .{ }^{\mathrm{a}-\mathrm{d}}$ The means in a graph not sharing superscript significantly differ $(P<0.05)$. Dpi, days postinfection. 

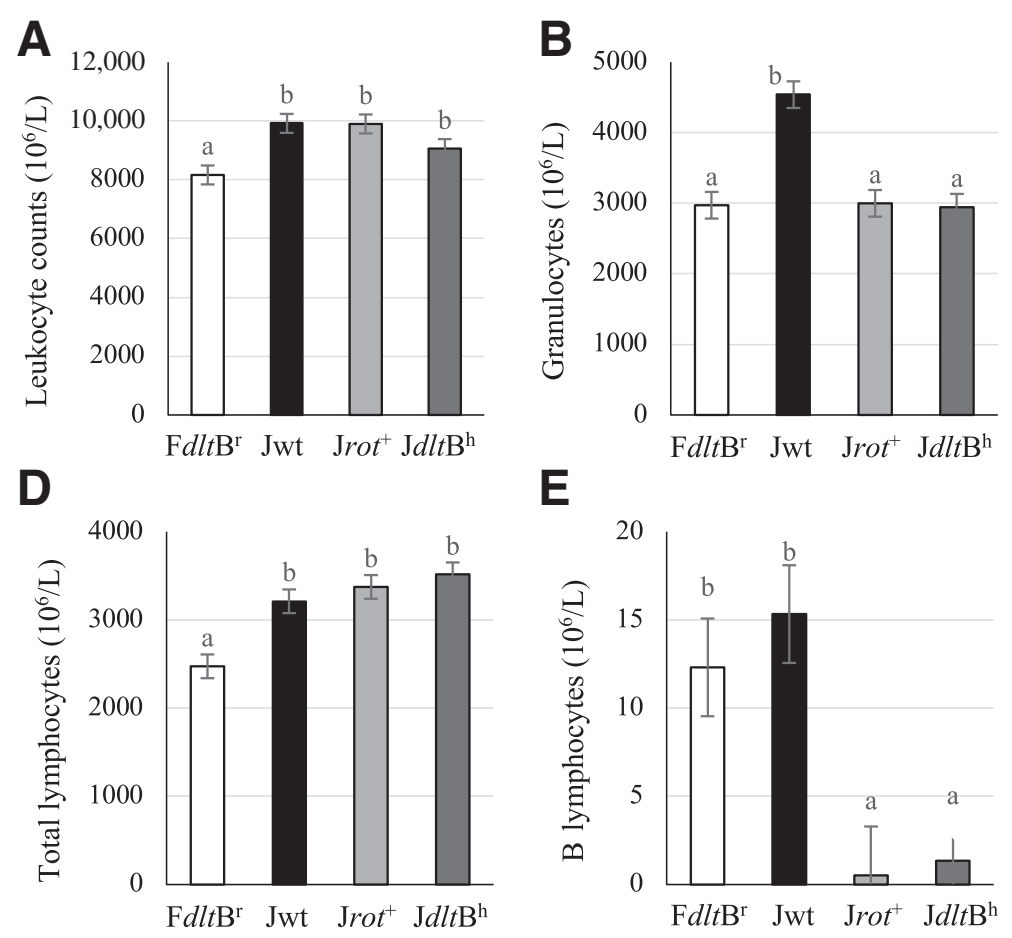

$\mathbf{E}$
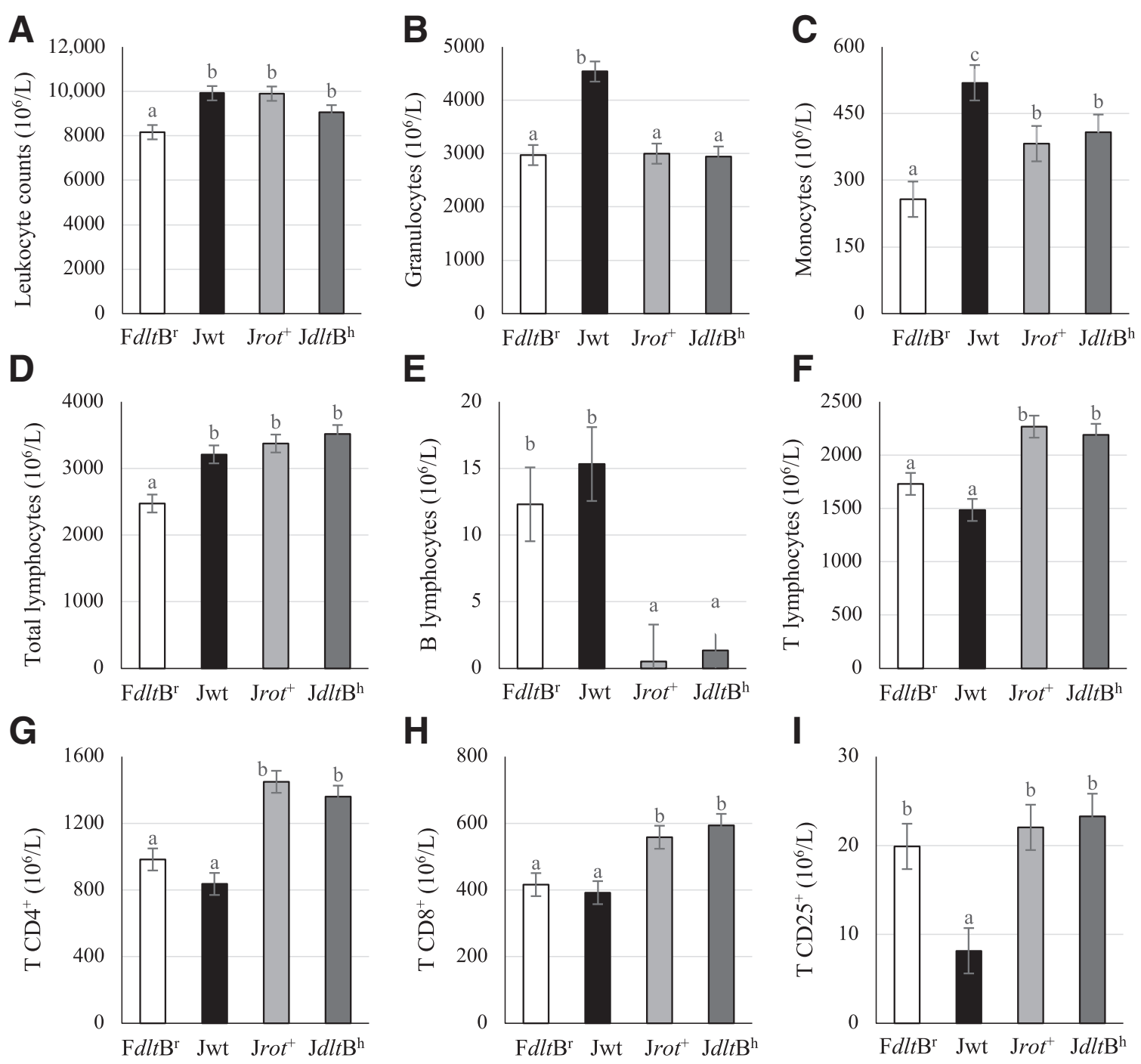

H
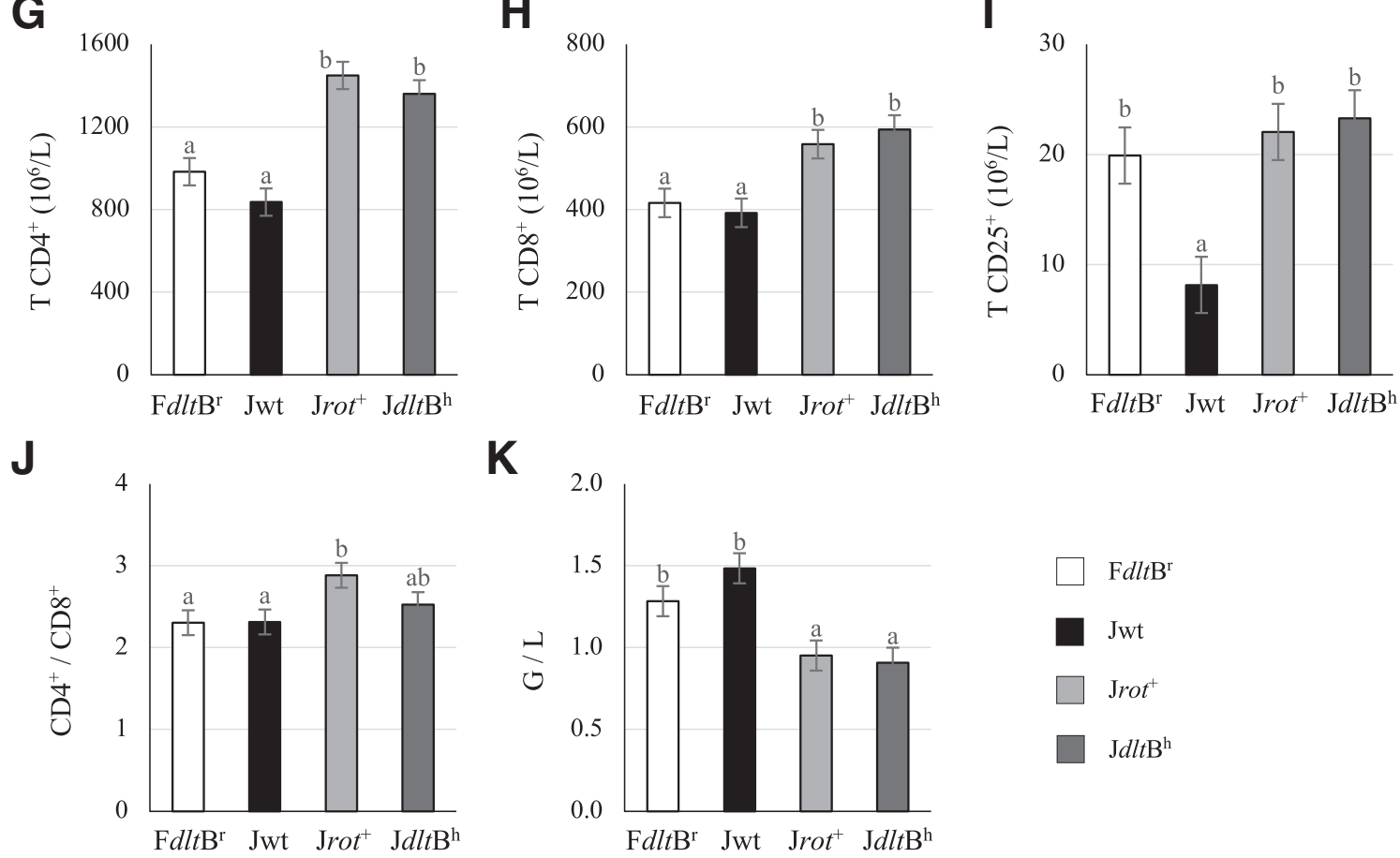

Figure 12 Effect of Staphylococcus aureus strains on leukocyte population counts $(\mathbf{A}-\mathbf{I})$ in peripheral blood and $\operatorname{CD} 4^{+} / \mathrm{CD} 8(\mathbf{J})$ and granulocyte/ lymphocyte $(\mathrm{G} / \mathrm{L} ; \mathbf{K})$ ratios after intradermal inoculation with strains $\mathrm{Fdlt}_{\mathrm{B}}{ }^{\mathrm{r}}$, Jwt, Jrot ${ }^{+}$, and $\mathrm{Jdlt} \mathrm{B}^{\mathrm{h}} . n=30$ animals per strain $(\mathbf{A}-\mathbf{K}){ }^{\mathrm{a}-\mathrm{c}}{ }^{\mathrm{T}} \mathrm{The}$ means in a same graph not sharing superscript significantly differ $(P<0.05)$.

higher on 3 and $7 \mathrm{dpi}$, respectively. The lesions produced by the inoculation of $\mathrm{Jrot}^{+}$were characterized mainly by the absence of $\mathrm{T}$ lymphocytes $\left(\mathrm{CD}^{+}\right)$on all of the analyzed days and high number of B lymphocytes on $3 \mathrm{dpi}$, similar to Jwt. The lesions produced by the inoculation of $\mathrm{J} d l t \mathrm{~B}^{\mathrm{h}}$ were characterized mainly by the absence of $\mathrm{B}$ lymphocytes $\left(\mathrm{CD} 79 \alpha^{+}\right)$on all of the sampling times.
Flow Cytometer Results

The differences detected in the peripheral blood cell counts in the rabbits infected by $S$. aureus $\mathrm{F} d l t \mathrm{~B}^{\mathrm{r}}$, Jwt, $\mathrm{Jrot}^{+}$, and $\mathrm{J} d l t \mathrm{~B}^{\mathrm{h}}$ are shown in Figure 12. The animals infected by the $\mathrm{F} d t \mathrm{~B}^{\mathrm{r}}$ strain had fewer leukocytes $(-15.2 \%)$ and total lymphocytes $(-26.5 \%)$ than $\mathrm{Jwt}, \mathrm{Jrot}^{+}$, and $\mathrm{J} d l t \mathrm{~B}^{\mathrm{h}}$, with similar counts to one another (Figure 12, A and D). 
In the $\mathrm{B}, \mathrm{T}, \mathrm{CD} 4^{+}$, and $\mathrm{CD} 8^{+}$lymphocyte counts, no significant differences were observed between the rabbits inoculated with the $\mathrm{F} d l t \mathrm{~B}^{\mathrm{r}}$ strain and Jwt. Both animal groups had more B lymphocytes and fewer $\mathrm{T}, \mathrm{CD} 4^{+}$, and $\mathrm{CD}^{+}$lymphocytes than $\mathrm{Jrot}^{+}$and $\mathrm{J} d l t \mathrm{~B}^{\mathrm{h}}$ (Figure 12, E-H). $\mathrm{F} d l t \mathrm{~B}^{\mathrm{r}}$ and Jwt strains had a lower $\mathrm{CD} 4^{+} / \mathrm{CD}^{+}$ratio than Jrot $^{+}$(Figure 12J).

The animals infected by the mutant strains presented fewer granulocytes $(-34.5 \% ; P<0.05)$ and monocytes than those inoculated with Jwt (Figure 12, B and C). For monocytes, the animals inoculated with $\mathrm{Jrot}^{+}$and $\mathrm{J} d l t \mathrm{~B}^{\mathrm{h}}$ had an intermediate level $\left(-23.9 \% \mathrm{Jrot}^{+}\right.$and $\mathrm{J} d l t \mathrm{~B}^{\mathrm{h}}$ and $\left.-50.5 \% \mathrm{~F} l t \mathrm{~B}^{\mathrm{r}} ; P<0.05\right)$ (Figure $12 \mathrm{C}$ ). The $\mathrm{CD} 25^{+}$lymphocytes showed significantly higher counts in the animals infected by mutant strains $\mathrm{F} d l t \mathrm{~B}^{\mathrm{r}}$, $\mathrm{Jrot}^{+}$, and $\mathrm{J} d l t \mathrm{~B}^{\mathrm{h}}(50.4 \%, 61.2 \%$, and $65 \%$, respectively; $P<0.05$ ) than the rabbits infected by Jwt (Figure 12I). Finally, strains $\mathrm{F} d l t \mathrm{~B}^{\mathrm{r}}$ and Jwt had a higher granulocyte/lymphocyte ratio than $\mathrm{Jrot}^{+}$and $\mathrm{J} d l t \mathrm{~B}^{\mathrm{h}}$ (Figure 12K).

\section{Discussion}

Staphylococcal infections have spread worldwide in hospitals and communities in the last decades of the 20th Century, are multiresistant to antibiotic strains (especially methicillin-resistant $S$. aureus), and have become a major health challenge in all industrialized countries today. ${ }^{1}$ This problem has also extended to farm animals since 2005, when a livestock-associated methicillin-resistant $S$. aureus was isolated in pigs ${ }^{32}$ and extended to other animal species, including horses, cattle, poultry, or rabbits. ${ }^{33-35}$ Among the wide range of diseases associated with community infections that can be caused by $S$. aureus, skin and soft tissue infections are one of the most worrying because they can be a life-threatening process if they are complicated. ${ }^{4-9}$ Skin and soft tissue infections are characterized by the formation of bacterial abscesses in dermis, epidermis, or subcutaneous tissues. $^{1}$

In this context, it would be valuable to have a definitive experimental model that allows the pathogenesis of the infection to be studied or new therapeutic tools to be tested. Mice are commonly used for staphylococcal colonization and infection models, mainly for their small size, easy handling, and the abundance of research facilities compared with other larger species, like rabbits. ${ }^{16,36}$ However, these characteristics alone do not justify their use if the results are not truly representative. Traditionally, rabbit models of $S$. aureus infection have been considered optimal to investigate virulence and host-pathogen interactions, mainly because of certain similarities with the human species: i) similar thickness of their $\operatorname{skin}^{14,15}$; ii) similar immune response to infection ${ }^{16}$; and iii) both are naturally infected by $S$. aureus. For these reasons, we present a rabbit model because this animal species has its own adapted $S$. aureus strains, ${ }^{12,35,36}$ which easily allow to naturally study staphylococcal infections.
The experimental model was respectful of animal health as no animal displayed fever, loss of weight, septicemia, or external signs of disease, except for the expected local lesions at inoculation points. The model was reproducible, and previously described characteristic abscesses developed, ${ }^{37}$ but it also provided more detailed characteristics of lesion development over time than previously described. Although lesions were observed at all the studied time points, staphylococcal infection with a Jwt strain was evaluated with this model at three key time points, 1,3 , and 7 dpi. On $1 \mathrm{dpi}$, it was possible to observe small slightly raised reddish papules that were histologically characterized by the presence of acute vascular changes, small organized abscesses, sometimes with inner bacteria and surrounded by heterophils, that were diffusely distributed. One interesting finding was the sharp and one-time increase in the CD25 lymphocytes 12 hours before $(0.5 \mathrm{dpi})$. As these cells are considered T-regulatory cells, ${ }^{38}$ it would be interesting to verify to what extent the inflammatory response described in this intradermal infection is modulated by this cell population. T-regulatory cells are essential for preventing exacerbation of inflammatory response, but they have been found to lose their activity in the presence of $S$. aureus, as shown in children with atopic skin. ${ }^{39,40}$ On $3 \mathrm{dpi}$, superficial lesions lost their initial reddish color and became nodular lesions, and it was when they acquired their largest volume and had more bacteria. Microscopically, vascular changes increased in severity, and abscesses were surrounded by an eosinophilic necrotic layer in whose core Splendore-Hoeppli phenomena with inner bacteria were observed. Surrounding the abscess, there were increasing numbers of $\mathrm{T}$ lymphocytes $\left(\mathrm{CD}^{+}\right)$and macrophages $\left(\mathrm{RAM} 11^{+}\right)$compared with previous days, which remained high until $21 \mathrm{dpi}$, and were related with the increased number of blood monocytes and lymphocytes on $2 \mathrm{dpi}$, as previously described. ${ }^{41}$ On $7 \mathrm{dpi}$, $50 \%$ of the nodules experienced epidermal necrosis and opened, which triggered a discharge of pus and a reduction in both lesion size and the number of isolated bacteria. These epidermal lesions generated a new inflammatory episode, peripherally characterized by an increased number of heterophils in blood, which lasted until 14 dpi. In histologic terms, lesions reached their most severe point at all of the studied locations, with several Splendore-Hoeppli phenomena surrounded by many $\mathrm{T}$ lymphocytes $\left(\mathrm{CD}^{+}\right)$, plasma cells $\left(\mathrm{IgG}^{+}\right)$, and macrophages $\left(\mathrm{RAM} 11^{+}\right)$.

Therefore, by studying on 1 and $3 \mathrm{dpi}$, it would be possible to evaluate the acute inflammatory response, as well as the subacute inflammatory reaction and initial repair mechanisms on $7 \mathrm{dpi}$, which could be useful for most experimental studies on this topic. For chronic inflammation studies, other later study times (eg, 14, 21, or $28 \mathrm{dpi}$ ) would be more recommendable, when regenerative modifications were predominant (re-epithelization and acanthosis in the epidermis, granulation tissue and fibrosis in the dermis, and regeneration of the cutaneous muscle), and when blood monocytes (2 to $14 \mathrm{dpi}$ ) and tissue lymphocytes and 
macrophages (3 to $21 \mathrm{dpi}$ ) significantly increased, as previously described for chronic inflammatory processes. ${ }^{41}$

Several findings in human and animal models have suggested a primarily role for T cells in immunity to $S$. aureus skin infections by enhancing the recruitment of phagocytes. $^{42-44}$ Staphylococcus aureus antigens induce different type 1, 2, and 17 helper T-cell pathways. Therefore, the role of T cells in immunity to $S$. aureus skin infections likely involves multiple T-cell effector cytokines. As there is a paucity of reagents available to analyze panels of rabbit immune mediators, in this work it was not possible to accurately characterize the immune response type, but significant differences were observed in some cytokines after the Jwt strain inoculation. Significant changes were found in cytokines IFN- $\gamma$ and IL-18. Cytokine IFN- $\gamma$ is a potent activator of monocytes by increasing their phagocytic activity in tissues. ${ }^{41}$ Despite this cytokine increasing on 21 dpi, it became significant only on 0.5 dpi. IL-18 has been associated with atopic dermatitis expelled by $S$. aureus. ${ }^{45}$ Although $S$. aureus is capable of causing the release of IL-18 from keratinocytes, ${ }^{46}$ in this work, an increase was detected only in plasma, but not in skin. An increase in IL-4 in plasma and skin was noted, especially after 21 days. Staphylococcus aureus is able to inhibit the response of Tcell responses and to induce type 2 helper T-cell responses by producing IL- $4^{47}$ in relation to chronic inflammations. ${ }^{41}$

The difficulty in developing an experimental model that reproduces an immune response under natural conditions might be related to the effect of the origin of both the bacteria and animal species used as hosts on the infective dose. $^{48}$ In general, a higher dose is necessary to infect animals with the $S$. aureus strains obtained from a different species (eg, human bacteria community-acquired methicillin-resistant $S$. aureus USA300 in mice: $\geq 5.0 \times 10^{8}$ $\mathrm{CFUs}^{49}$ ). In such cases, animal models can be unreliable predictors of either the potential success of therapeutic or preventive interventions or the roles played by specific determinants of bacterial virulence in infection. ${ }^{2}$ Only a few standardized studies about the number of bacteria needed to begin infection can be found. ${ }^{50}$ Schmid-Hempel and Frank $^{50}$ proposed an infective $S$. aureus dose of $10^{5}$ to $10^{6}$ bacteria and classified it as a high-infective dose microorganism. In fact, it has been reported that inoculation with $<5 \times 10^{8} \mathrm{CFUs}$ of USA300 does not result in reproducible abscesses in rabbits. ${ }^{17,49}$ The results from the current study agree with this observation when human strains (not adapted to rabbit) were used, but disagree when rabbit strains, which can generate dermal lesions with only 300 CFUs by simulating natural infection, were employed. Moreover, when high doses of human strain Fwt ( $>10^{6}$ CFUs) were used with this model, the generated lesions differed from those observed for low doses of rabbit wild-type strains: lesions rapidly evolved and skin necrotic phenomena (dermonecrosis) predominated and, in histologic terms, the presence of thrombosis and the absence of Splendore-Hoeppli phenomena or a peripheral eosinophilic layer were observed on
3 dpi (J.M.C., unpublished data) (Supplemental Figure S1). Similar results have been reported by other authors, ${ }^{17}$ who recognized that using such high doses would result in rapidly progressive infection, which would vastly differ from that typically encountered in clinical situations, and the results could not be extrapolated to humans. These skin necrotic phenomena are probably caused by the mass production of bacterial toxins, which is far removed from what actually happens in natural infection. This hypothesis would support the results observed in the present study with the mutants ( $\mathrm{J} \Delta \operatorname{coa} \Delta v w b, \mathrm{~J} \Delta h l a, \mathrm{~J} \Delta p \operatorname{sm} \alpha$, and $\mathrm{J} \Delta a g r)$ employed to evaluate their contribution to the development and characteristics of abscesses. Studies performed in rabbits, and mainly in mice, using $\Delta h l a$ mutants have resulted in reduced virulence in animal models of dermonecrotic skin infection. Moreover, immunization with alphahemolysin-specific antisera significantly reduces the size of skin lesions and prevents dermonecrosis, ${ }^{11,17,51,52}$ and similar results have been obtained with mutants in $p s m \alpha .^{53}$ Surprisingly, when the herein described skin infection model was used, mutants $\Delta$ hla and $\Delta p s m \alpha$ produced the same percentage of lesions as the wild-type strain, and even became macroscopically more severe for $\Delta h l a$ regarding Jwt. Toxins and other virulence factors, such as $\alpha$-toxin, phenol-soluble modulin $\alpha$ (PSM- $\alpha$ ), and PSM- $\beta$, are produced as a result of increased bacterial population density and environmental conditions, partly under a quorumsensing control through different regulators. ${ }^{54-56}$ Therefore, the effect observed in previous works with either the $\Delta h l a$ or the $\Delta p s m \alpha$ mutant could be due to the use of high doses of bacteria to produce lesions, which would favor the rapid production of numerous toxins, and would make the effect of the toxin more evident. The absence of an effect on the $\Delta a g r$ mutant would follow the same hypothesis. In $S$. aureus, most quorum-sensing components are coded by the accessory gene regulator (Agr) system. This regulation is important for acute disease to develop, ${ }^{37}$ but we showed that Agr was dispensable in intradermal infections of rabbits. Agr suppresses the expression of the repressor of toxins (rot) at a high cell density, ${ }^{57}$ which could also influence the virulence of the $\Delta a g r$ mutant. However, rabbit strains have a natural loss-of-function mutation in rot, which increases the severity and infectivity of the rabbit strain. This could justify the $F d l t \mathrm{~B}^{\mathrm{r}}$ strain (with functional rot) producing milder lesions than the wild-type strain.

A histologic characteristic observed from 3 dpi in the lesions produced by the strain wild type was an eosinophilic necrotic layer in whose core Splendore-Hoeppli phenomena with inner bacteria were observed. These phenomena have been previously described as an electron-dense, granular, and amorphous pseudocapsule. ${ }^{37,58,59}$ This pseudocapsule constitutes a barrier for immune cells by preventing them from penetrating the staphylococcal abscess community, where bacteria replicate without interference and are partially composed of prothrombin and fibrinogen that separate bacteria from leukocytes. ${ }^{37,58,59}$ As the $\Delta$ coa $\Delta v w b$ 
mutant was able to produce lesions like Jwt, the absence of these two clotting factors secreted by $S$. aureus does not seem essential for abscesses to develop in rabbits. Despite all of the employed mutants ( $\mathrm{J} \Delta \operatorname{coa} \Delta v w b, \mathrm{~J} \Delta h l a, \mathrm{~J} \Delta p s m \alpha$, and $\mathrm{J} \Delta a g r$ ) being able to generate similar lesions to the wild type in size and degree of severity terms, albeit in different percentages, they all triggered a delay in the evolution of histologic lesions compared with the wild type. This could indicate that their mutations affected abscess development, but other factors also had to be involved in their development. More studies are needed to learn the specific role of these and other virulence factors in $S$. aureus infection development.

The utility of this model was corroborated when a human strain, modified to infect rabbits (rabbitized), was inoculated $\left(\mathrm{F} d l t \mathrm{~B}^{\mathrm{r}}\right)$. This adaptation to the host seemed essential because neither macroscopic nor microscopic lesions developed when the human Fwt strain was inoculated with $300 \mathrm{CFUs},{ }^{13}$ whereas the modified strain $\left(\mathrm{F} d l t \mathrm{~B}^{\mathrm{r}}\right)$ was able to produce similar lesions to the wild strain adapted to rabbit (Jwt).

Finally, although mouse models have been presented as the gold standard to study staphylococcal infections in humans, along with their therapy and prevention, ${ }^{60}$ we propose a low-infective-dose rabbit model as a more representative and realistic model, in which the effect of both homologous and heterologous strains can be proved.

\section{Acknowledgments}

We thank José R. Penadés for support with the development of this work and comments on the manuscript and Laura Núñez for excellent technical assistance.

\section{Author Contributions}

L.S., J.M.C., and D.V. conceived the study; J.J.P., A.A.-B, J.M.C., and D.V. analyzed the results; L.S., J.M.C., and D.V. acquired funding; A.M.-S., M.P., L.S., S.P.-F., E.M., A.G.-Q., J.J.P., A.A.-B, A.B., J.M.C., and D.V. performed experiments; J.M.C. provided project administration; J.M.C. and D.V. supervised the study; A.M.-S., A.A.-B., J.M.C., and D.V. wrote the manuscript; A.A.-B., J.J.P, J.M.C., and D.V. reviewed and edited the manuscript; J.M.C. and D.V. are the guarantors of this work and, as such, had full access to all of the data in the study and take responsibility for the integrity of the data and the accuracy of the data analysis.

\section{Supplemental Data}

Supplemental material for this article can be found at http://doi.org/10.1016/j.ajpath.2020.01.019.

\section{References}

1. Kobayashi SD, Malachowa N, DeLeo FR: Pathogenesis of Staphylococcus aureus abscesses. Am J Pathol 2015, 185:1518-1527

2. Lowy FD: How Staphylococcus aureus adapts to its host. N Engl J Med 2011, 364:1987-1990

3. Peschel A, Otto M: Phenol-soluble modulins and staphylococcal infection. Nat Vet Microbiol 2013, 11:667-673

4. Eiff von C, Becker K, Machka K, Stammer H, Peters G: Nasal carriage as a source of Staphylococcus aureus bacteremia. N Engl J Med 2001, 344:11-16

5. Said-Salim B, Dunman PM, McAleese FM, Macapagal D, Murphy E, McNamara PJ, Arvidson S, Foster TJ, Projan SJ, Kreiswirth BN: Global regulation of Staphylococcus aureus genes by Rot. J Bacteriol 2003, 185:610-619

6. Gao J, Stewart GC: Regulatory elements of the Staphylococcus aureus protein A (Spa) promoter. J Bacteriol 2004, 186:3738-3748

7. Fridkin SK, Hageman JC, Morrison M, Sanza LT, Como-Sabetti K, Jernigan JA, Harriman K, Harrison LH, Lynfield R, Farley MM; Active Bacterial Core Surveillance Program of the Emerging Infections Program Network: Methicillin-resistant Staphylococcus aureus disease in three communities. N Engl J Med 2005, 352: $1436-1444$

8. DeLeo FR, Otto M, Kreiswirth BN, Chambers HF: Communityassociated methicillin-resistant Staphylococcus aureus. Lancet 2010, 375:1557-1568

9. Talan DA, Krishnadasan A, Gorwitz RJ, Fosheim GE, Limbago B, Albrecht V, Moran GJ; EMERGEncy ID Net Study Group: Comparison of Staphylococcus aureus from skin and soft-tissue infections in US emergency department patients, 2004 and 2008. Clin Infect Dis 2011, 53:144-149

10. Bae I-G, Tonthat GT, Stryjewski ME, Rude TH, Reilly LF, Barriere SL, Genter FC, Corey GR, Fowler VG: Presence of genes encoding the panton-valentine leukocidin exotoxin is not the primary determinant of outcome in patients with complicated skin and skin structure infections due to methicillin-resistant Staphylococcus aureus: results of a multinational trial. J Clin Microbiol 2009, 47: 3952-3957

11. Kennedy AD, Wardenburg JB, Gardner DJ, Long D, Whitney AR, Braughton KR, Schneewind O, DeLeo FR: Targeting of alpha-hemolysin by active or passive immunization decreases severity of USA300 skin infection in a mouse model. J Infect Dis 2010, 202: 1050-1058

12. Viana D, Selva L, Segura P, Penadés JR, Corpa JM: Genotypic characterization of Staphylococcus aureus strains isolated from rabbit lesions. Vet Microbiol 2007, 121:288-298

13. Viana D, Comos MA, McAdam PR, Ward MJ, Selva L, Guinane CM, González-Muñoz BM, Tristan A, Foster SJ, Fitzgerald JR, Penadés JR: A single natural nucleotide mutation alters bacterial pathogen host tropism. Nat Genet 2015, 47:361-366

14. Oznurlu Y, Celik I, Sur E, Telatar T, Ozparlak H: Comparative skin histology of the white New Zealand and angora rabbits: histometrical and immunohistochemical evaluations. JAVA 2009, 8: 1694-1701

15. Jung EC, Maibach HI: Animal models for percutaneous absorption. J Appl Toxicol 2015, 35:1-10

16. Malachowa N, Kobayashi SD, Porter AR, Braughton KR, Scott DP, Gardner DJ, Missiakas DM, Schneewind O, DeLeo FR: Contribution of Staphylococcus aureus coagulases and clumping factor A to abscess formation in a rabbit model of skin and soft tissue infection. PLoS One 2016, 11:e158293-14

17. Le VTM, Tkaczyk C, Chau S, Rao RL, Dip EC, Pereira-Franchi EP, Cheng L, Lee S, Koelkebeck H, Hilliard JJ, Yu XQ, Datta V, Nguyen V, Weiss W, Prokai L, O'Day T, Stover CK, Sellman BR, Diep BA: Critical role of alpha-toxin and protective effects of its neutralization by a human antibody in acute bacterial skin and skin 
structure infections. Antimicrob Agents Chemother 2016, 60 $5640-5648$

18. Otto M: Staphylococcus aureus toxins. Curr Opin Microbiol 2014, $17: 32-37$

19. Collins LV, Kristian SA, Weidenmaier C, Faigle M, van Kessel KPM, van Strijp JAG, Götz F, Neumeister B, Peschel A: Staphylococcus aureus strains lacking D-alanine modifications of teichoic acids are highly susceptible to human neutrophil killing and are virulence attenuated in mice. J Infect Dis 2002, 186:214-219

20. Novick RP: Genetic systems in staphylococci. Meth Enzymol 1991, 204:587-636

21. Lindsay JA, Ruzin A, Ross HF, Kurepina N, Novick RP: The gene for toxic shock toxin is carried by a family of mobile pathogenicity islands in Staphylococcus aureus. Mol Microbiol 1998, 29:527-543

22. Ausubel FM, Brent R, Kingston RE, Moore DD, Seidman JG, Smith JA, Struhl K: Current Protocols in Molecular Biology. New York, NY, John Wiley \& Sons, 1990

23. Arnaud M, Chastanet A, Débarbouillé M: New vector for efficient allelic replacement in naturally nontransformable, low-GC-content, gram-positive bacteria. Appl Environ Microbiol 2004, 70:6887-6891

24. Ubeda C, Maiques E, Knecht E, Lasa I, Novick RP, Penadés JR: Antibiotic-induced SOS response promotes horizontal dissemination of pathogenicity island-encoded virulence factors in staphylococci. Mol Microbiol 2005, 56:836-844

25. Li M, Cheung GYC, Hu J, Wang D, Joo H-S, DeLeo FR, Otto M: Comparative analysis of virulence and toxin expression of global community-associated methicillin-resistant Staphylococcus aureus strains. J Infect Dis 2010, 202:1866-1876

26. Bunce C, Wheeler L, Reed G, Musser J, Barg N: Murine model of cutaneous infection with gram-positive cocci. Infect Immun 1992, 60: $2636-2640$

27. Voyich JM, Otto M, Mathema B, Braughton KR, Whitney AR, Welty D, Long RD, Dorward DW, Gardner DJ, Lina G, Kreiswirth BN, DeLeo FR: Is panton-valentine leukocidin the major virulence determinant in community-associated methicillin-resistant Staphylococcus aureus disease? J Infect Dis 2006, 194:1761-1770

28. Jeklova E, Leva L, Faldyna M: Lymphoid organ development in rabbits: major lymphocyte subsets. Dev Comp Immunol 2007, 31 : 632-644

29. Guerrero I, Ferrian S, Blas E, Pascual JJ, Cano JL, Corpa JM: Evolution of the peripheral blood lymphocyte populations in multiparous rabbit does with two reproductive management rhythms. Vet Immunol Immunopathol 2011, 140:75-81

30. Hulstaert F, Hannet I, Deneys V, Munhyeshuli V, Reichert T, De Bruyere M, Strauss K: Age-related changes in human blood lymphocyte subpopulations, II: varying kinetics of percentage and absolute count measurements. Clin Immunol Immunopathol 1994, 70:152-158

31. McCullagh P, Nelder JA: Generalized Linear Models, ed 2. Boca Raton, FL: Chapman \& Hall/CRC, 1989

32. Armand-Lefevre L, Ruimy R, Andremont A: Clonal comparison of Staphylococcus aureus isolates from healthy pig farmers, human controls, and pigs. Emerg Infect Dis 2005, 11:711-714

33. Graveland H, Duim B, van Duijkeren E, Heederik D, Wagenaar JA: Livestock-associated methicillin-resistant Staphylococcus aureus in animals and humans. Int J Med Microbiol 2011, 301:630-634

34. Aires-de-Sousa M: Methicillin-resistant Staphylococcus aureus among animals: current overview. Clin Microbiol Infect 2017, 23: $373-380$

35. Moreno-Grúa E, Pérez-Fuentes S, Muñoz-Silvestre A, Viana D, Fernández-Ros AB, Sanz-Tejero C, Corpa JM, Selva L: Characterization of livestock-associated methicillin-resistant Staphylococcus aureus isolates obtained from commercial rabbitries located in the Iberian peninsula. Front Microbiol 2018, 9:1812

36. Viana D, Selva L, Callanan JJ, Guerrero I, Ferrian S, Corpa JM: Strains of Staphylococcus aureus and pathology associated with chronic suppurative mastitis in rabbits. Vet J 2011, 190: 403-407
37. Cheng AG, DeDent AC, Schneewind O, Missiakas D: A play in four acts: Staphylococcus aureus abscess formation. Trends Microbiol 2011, 19:225-232

38. Chen X, Du Y, Lin X, Qian Y, Zhou T, Huang Z: CD4+CD25+ regulatory T cells in tumor immunity. Int Immunopharmacol 2016, $34: 244-249$

39. Bekeredjian-Ding I: Deciphering the significance of the T-cell response to Staphylococcus aureus. Future Microbiol 2017, 12 $1023-1026$

40. Laborel-Préneron E, Bianchi P, Boralevi F, Lehours P, Fraysse F, Morice-Picard F, Sugai M, Sato'o Y, Badiou C, Lina G, Schmitt AM, Redoulès D, Casas C, Davrinche C: Effects of the Staphylococcus aureus and Staphylococcus epidermidis secretomes isolated from the skin microbiota of atopic children on CD4+ T cell activation. PLoS One 2015, 10:e0141067-16

41. Ackermann MR: Chapter 3 - inflammation and healing. Edited by Zachary JF. In Pathologic Basis of Veterinary Disease. ed 6. St. Louis, MO: Elsevier Inc, 2017. pp. 73-131.e2

42. Krishna S, Miller LS: Innate and adaptive immune responses against Staphylococcus aureus skin infections. Semin Immunopathol 2011, 34:261-280

43. Liu Q, Mazhar M, Miller LS: Immune and inflammatory reponses to Staphylococcus aureus skin infections. Curr Dermatol Rep 2018, 7: 338-349

44. Miller LS, Cho JS: Immunity against Staphylococcus aureus cutaneous infections. Nat Rev Immunol 2011, 11:505-518

45. Terada M, Tsutsui H, Imai Y, Yasuda K, Mizutani H, Yamanishi K, Kubo M, Matsui K, Sano H, Nakanishi K: Contribution of IL-18 to atopic-dermatitis-like skin inflammation induced by Staphylococcus aureus product in mice. Proc Natl Acad Sci U S A 2006, 103 8816-882

46. Syed AK, Reed TJ, Clark KL, Boles BR, Kahlenberg JM: Staphylococcus aureus phenol-soluble modulins stimulate the release of proinflammatory cytokines from keratinocytes and are required for induction of skin inflammation. Infect Immun 2015, $83: 3428-3437$

47. Laouini D, Kawamoto S, Yalcindag A, Bryce P, Mizoguchi E, Oettgen H, Geha RS: Epicutaneous sensitization with superantigen induces allergic skin inflammation. J Allergy Clin Immunol 2003, 112:981-987

48. Holtfreter S, Radcliff FJ, Grumann D, Read H, Johnson S, Monecke S, Ritchie S, Clow F, Goerke C, Bröker BM, Fraser JD Wiles S: Characterization of a mouse-adapted Staphylococcus aureus strain. PLoS One 2013, 8:e71142

49. Kobayashi SD, Malachowa N, Whitney AR, Braughton KR, Gardner DJ, Long D, Bubeck Wardenburg J, Schneewind O, Otto M, DeLeo FR: Comparative analysis of USA300 virulence determinants in a rabbit model of skin and soft tissue infection. J Infect Dis 2011, 204:937-941

50. Schmid-Hempel P, Frank SA: Pathogenesis, virulence, and infective dose. PLoS Pathog 2007, 3:1372-1373

51. Inoshima N, Wang Y, Bubeck Wardenburg J: Genetic requirement for ADAM10 in severe Staphylococcus aureus skin infection. J Invest Dermatol 2012, 132:1513-1516

52. Tkaczyk C, Hamilton MM, Datta V, Yang XP, Hilliard JJ, Stephens GL, Sadowska A, Hua L, O’Day T, Suzich J, Stover CK, Sellman BR: Staphylococcus aureus alpha toxin suppresses effective innate and adaptive immune responses in a murine dermonecrosis model. PLoS One 2013, 8:e75103-e75112

53. Wang R, Braughton KR, Kretschmer D, Bach T-HL, Queck SY, Li M, Kennedy AD, Dorward DW, Klebanoff SJ, Peschel A, DeLeo FR, Otto M: Identification of novel cytolytic peptides as key virulence determinants for community-associated MRSA. Nat Med 2007, 13:1510-1514

54. Recsei P, Kreiswirth B, O'Reilly M, Schlievert P, Gruss A, Novick RP: Regulation of exoprotein gene expression in Staphylococcus aureus by agar. Mol Gen Genet 1986, 202:58-61 
55. Rainard P, Gitton C, Chaumeil T, Fassier T, Huau C, Riou M, TosserKlopp G, Krupova Z, Chaize A, Gilbert FB, Rupp R, Martin P: Host factors determine the evolution of infection with Staphylococcus aureus to gangrenous mastitis in goats. Vet Res 2018, 49:72

56. Salam AM, Quave CL: Targeting virulence in Staphylococcus aureus by chemical inhibition of the accessory gene regulator system in vivo. mSphere 2018, 3:1193

57. Haag AF, Bagnoli F: The role of two-component signal transduction systems in Staphylococcus aureus virulence regulation. Curr Top Microbiol Immunol 2017, 409:145-198
58. Cheng AG, Kim HK, Burts ML, Krausz T, Schneewind O, Missiakas DM: Genetic requirements for Staphylococcus aureus abscess formation and persistence in host tissues. FASEB J 2009, 23: 3393-3404

59. Cheng AG, McAdow M, Kim HK, Bae T, Missiakas DM, Schneewind O: Contribution of coagulases towards Staphylococcus aureus disease and protective immunity. PLoS Pathog 2010, 6:e1001036

60. Kim HK, Missiakas D, Schneewind O: Mouse models for infectious diseases caused by Staphylococcus aureus. J Immunol Methods 2014, 410:88-99 